\title{
Dynamical locality of the free scalar field
}

\author{
Christopher J. Fewster ${ }^{1 *}$ and Rainer Verch2计 \\ ${ }^{1}$ Department of Mathematics, University of York, Heslington, York YO10 5DD, U.K. \\ 2 Institut für Theoretische Physik, Universität Leipzig, 04009 Leipzig, Germany
}

November 8, 2018

\begin{abstract}
Dynamical locality is a condition on a locally covariant physical theory, asserting that kinematic and dynamical notions of local physics agree. This condition was introduced in arXiv:1106.4785, where it was shown to be closely related to the question of what it means for a theory to describe the same physics on different spacetimes. In this paper, we consider in detail the example of the free minimally coupled Klein-Gordon field, both as a classical and quantum theory (using both the Weyl algebra and a smeared field approach). It is shown that the massive theory obeys dynamical locality, both classically and in quantum field theory, in all spacetime dimensions $n \geq 2$ and allowing for spacetimes with finitely many connected components. In contrast, the massless theory is shown to violate dynamical locality in any spacetime dimension, in both classical and quantum theory, owing to a rigid gauge symmetry. Taking this into account (equivalently, working with the massless current) dynamical locality is restored in all dimensions $n \geq 2$ on connected spacetimes, and in all dimensions $n \geq 3$ if disconnected spacetimes are permitted. The results on the quantized theories are obtained using general results giving conditions under which dynamically local classical symplectic theories have dynamically local quantizations.
\end{abstract}

\section{Introduction}

In the functorial description of locally covariant quantum field theory in curved spacetimes introduced by Brunetti, Fredenhagen and Verch (BFV) [8], a physical theory is described by a functor from a category of globally hyperbolic spacetimes to a category of $(C)^{*}$ algebras. This view-point has proved fruitful in various aspects of model-independent quantum field theory, e.g., the proof of the spin-statistics connection [43, analogues of the Reeh-Schlieder theorem [40, 14, superselection theory [10, 11, and the perturbative construction of interacting theories in curved spacetime [7, 32, 33]. Moreover, it has led to applications in cosmology [15, 17, 44] and constraints on Casimir energy densities in cavities of arbitrary geometry [26, 23].

The same formalism can be applied to branches of physics other than quantum field theory by suitable choice of the target category. As we have emphasized in [27] (see also [24]) the functorial framework allows us to analyse theories and relationships between

\footnotetext{
${ }^{*}$ E-mail: chris.fewster@york.ac.uk

${ }^{\dagger}$ E-mail: verch@itp.uni-leipzig.de
} 
theories at the level of the functors, rather than in individual spacetimes. In particular, we showed in [27] how one can even address questions such as whether a given theory can be regarded as representing the same physics in all spacetimes (SPASs). In particular, we gave a simple condition that should be obeyed by any reasonable notion of SPASs and showed that (a) it is not satisfied by the full class of locally covariant theories, but (b) it is satisfied by the subclass of theories obeying dynamical locality, which is free of the known pathologies associated with the full class.

The precise definition of dynamical locality will be reviewed in Sect. 22 roughly, it requires that kinematical and dynamical notions of localisation coincide for the theory. A variety of properties of dynamically local theories were studied in [27], suggesting that the property is of independent interest in locally covariant physics. For instance, it permits a general proof of the impossibility of selecting a single 'natural' state in each spacetime for any nontrivial dynamically local theory that reduces to a Haag-Kastler or Wightman theory in Minkowski space, with the supposed natural state as the vacuum (see [27, §6.3]). Previous results on this question were confined to free models, and even there were not absolutely complete arguments.

The present paper provides examples to illustrate the theory developed in [27]. In particular, we will show that the massive minimally coupled Klein-Gordon theory obeys the dynamical locality condition, but that the massless minimally coupled theory does not, unless it is formulated as a theory of currents. This property holds for both the classical and quantized theories, with quantization performed either using a $*$-algebra of smeared fields, or the Weyl $C^{*}$-algebra approach. Our results on the quantized theories are established as special cases of general results, which we prove, showing how dynamically local classical theories (valued in a category of symplectic spaces) have dynamically local quantizations under mild additional hypotheses.

The failure of dynamical locality in the massless case may be traced to the existence of the rigid gauge freedom $\varphi \mapsto \varphi+$ constant, which is normally ignored in treating this theory. Taking the gauge symmetry seriously, and quantizing the theory on the same lines as electromagnetism and related theories [20, 25, 38], we find that dynamical locality is restored with the sole exception of the theory in two-dimensional (potentially disconnected) spacetimes. Dynamical locality holds if one restricts to connected spacetimes. The significance of this sole exception is unclear and will provide the basis for further work. In a separate work [21] it will be shown that the nonminimally coupled Klein-Gordon theory obeys dynamical locality for any value of the mass (in this case there is no gauge freedom). Studies of other theories are under way.

The paper is structured as follows. We review the basic ideas and terminology of [27] in Sect. 2, and then discuss the dynamical locality of the classical minimally coupled field and massless current in Sects. 3 and 4. Our general results on quantization by the infinitesimal Weyl algebra and Weyl algebra approaches appear in Sects. 5 and 6. Remarks on related approaches appear in Sect. 7. The appendices give background on some multilinear algebra required in the body of the paper, and also establish the differentiability of the relative Cauchy evolution for the real scalar field. 


\section{Dynamical locality}

We briefly summarise the BFV approach to locally covariant physics, as elaborated in [27]. This is a framework for studying physics on fixed spacetimes, which are taken to be globally hyperbolic, but not necessarily connected. The fundamental definitions of category theory [35, 2] will be assumed, but some particular structures will be defined where necessary.

Spacetimes A spacetime of dimension $n \geq 2$ is a quadruple $(\mathcal{M}, \boldsymbol{g}, \mathfrak{o}, \mathfrak{t})$ such that $\mathcal{M}$ is a smooth, paracompact, orientable nonempty $n$-manifold with finitely many connected components, $\boldsymbol{g}$ is a smooth time-orientable metric of signature $+-\cdots-$ on $\mathcal{M}$, and $\mathfrak{o}$ and $\mathfrak{t}$ are choices of orientation and time-orientation respectively. A spacetime is said to be globally hyperbolic if it contains no closed causal curves and the intersection of the causal past and future of any pair of points is compact. It is sufficient that $\mathcal{M}$ contains a Cauchy surface, i.e., a subset met exactly once by every inextendible timelike curve in the spacetime. (A number of properties of globally hyperbolic spacetimes, and comments on different notions appearing in the literature may be found in Sect. 2 of [27].)

The globally hyperbolic spacetimes (of dimension $n$ ) form the objects of a category Loc. By definition, a morphism $\psi$ in Loc between $\boldsymbol{M}=(\mathcal{M}, \boldsymbol{g}, \mathfrak{o}, \mathfrak{t})$ and $\boldsymbol{M}^{\prime}=\left(\mathcal{M}^{\prime}, \boldsymbol{g}^{\prime}, \mathfrak{o}^{\prime}, \mathfrak{t}^{\prime}\right)$ is a smooth embedding (also denoted $\psi$ ) of $\mathcal{M}$ in $\mathcal{M}^{\prime}$ whose image is causally convex ${ }^{3}$ in $\boldsymbol{M}^{\prime}$ and such that $\psi^{*} \boldsymbol{g}^{\prime}=\boldsymbol{g}, \psi^{*} \mathfrak{o}^{\prime}=\mathfrak{o}$ and $\psi^{*} \mathfrak{t}^{\prime}=\mathfrak{t}$. Thus the embedding is isometric and respects orientation and time-orientation. The full subcategory of Loc with connected spacetimes as objects will be denoted $\operatorname{Loc}_{0}$.

Two particular classes of Loc and Loc $_{0}$ morphisms will be used extensively in what follows: canonical inclusions and Cauchy morphisms. Inclusions arise as follows. For any $\boldsymbol{M}$ in Loc (and hence $\operatorname{Loc}_{0}$ ) let $\mathscr{O}(\boldsymbol{M})$ be the set of open globally hyperbolic subsets of $\boldsymbol{M}$ with at most finitely many connected components all of which are mutually causally disjoint, and let $\mathscr{O}_{0}(\boldsymbol{M})$ be the set of connected open globally hyperbolic subsets of $\boldsymbol{M}$. For each $\boldsymbol{M}=(\mathcal{M}, \boldsymbol{g}, \mathfrak{o}, \mathfrak{t}) \in$ Loc, any nonempty $O \in \mathscr{O}(\boldsymbol{M})$ induces an object $\left.\boldsymbol{M}\right|_{O}=$ $\left(O,\left.\boldsymbol{g}\right|_{O},\left.\mathfrak{o}\right|_{O},\left.\mathfrak{t}\right|_{O}\right)$ of Loc, which we call the restriction of $\boldsymbol{M}$ to $O$, and the subset inclusion of $O$ in $\boldsymbol{M}$ induces a Loc-morphism $\iota_{\boldsymbol{M} ; O}:\left.\boldsymbol{M}\right|_{O} \rightarrow \boldsymbol{M}$ that we call a canonical inclusion. If $O \in \mathscr{O}_{0}(\boldsymbol{M})$ for $\boldsymbol{M} \in \operatorname{Loc}_{0}$ then $\iota_{\boldsymbol{M} ; O}$ is also a $\operatorname{Loc}_{0}$-morphism, provided $O$ is nonempty. A Cauchy morphism is a morphism $\psi: \boldsymbol{M} \rightarrow \boldsymbol{N}$ whose image $\psi(\boldsymbol{M})$ contains a Cauchy surface for $\boldsymbol{N}$.

Physical systems The categories $\mathrm{Loc}_{0}$ and Loc provide the arena for locally covariant physics. Physical systems themselves are described as objects in a category Phys, which is determined by the type of physical system under consideration. The general conditions imposed on Phys in [27] are:

- all morphisms in Phys are monic, i.e., $f \circ g=f \circ h$ implies $g=h$ for all $f, g, h$;

- there is an initial object, $\mathcal{I}$, i.e., for every object $A$ of Phys, there is a exactly one morphism from $\mathcal{I}$ to $A$, denoted $\mathcal{I}_{A}$;

\footnotetext{
${ }^{3}$ That is, it contains all causal curves of which it contains the endpoints.
} 
- it has equalizers, i.e., for any pair of morphisms $f, g: A \rightarrow B$ there is a morphism $h$ (the equalizer of $f$ and $g$ ) such that $f \circ h=g \circ h$ and such that if $k$ is any morphism such that $f \circ k=g \circ k$ then $k$ factorizes uniquely via $h$, i.e., $k=h \circ m$ for a unique morphism $m$ - we write $h \cong \mathrm{eq}(f, g)$;

- it has intersections and unions (see [18], and Appendix B to [27]).

Here, intersections and unions are not generally identical to the intersections and unions of set theory, but appropriate generalizations to the category in question, e.g., the 'union' in a category of vector spaces is determined by the linear span etc. Like equalizers, they are defined by universal properties, only up to isomorphism: if $f: A \rightarrow B$ is an intersection of the morphisms $f_{i}: A_{i} \rightarrow B$, and $g: A^{\prime} \rightarrow A$ is an isomorphism, then $f \circ g: A^{\prime} \rightarrow B$ is also an intersection of the $f_{i}$. We write $f \cong \bigwedge_{i} f_{i}$ in such circumstances, and likewise denote a union by $f \cong \bigvee_{i} f_{i}$. In concrete categories where the morphisms can be regarded as functions (with particular structure) and composition is composition of the functions, the 'up to isomorphism' nature of these definitions can largely be ignored, because the image of such a map is unchanged by precomposition with an isomorphism.

To illustrate the definitions, we give the examples which will be relevant to us in the present paper. In the quantum theory, we will use Alg, the category of unital complex $*$-algebras, with unit-preserving injective $*$-homomorphisms as the morphisms, and $\mathrm{C}^{*}$-Alg, the full subcategory of Alg consisting of $C^{*}$-algebras. The initial object is the algebra of complex numbers with 1 as unit and complex conjugation as the $*$-operation and (for $C^{*}$-Alg) the complex modulus as $C^{*}$-norm. Subobjects may be identified with $(C) *$-subalgebras; the intersection is the ordinary intersection of $(C) *$-subalgebras and the union is the $(C) *$-algebraic span; the equalizer of $\alpha, \beta: \mathcal{A} \rightarrow \mathcal{B}$ can be described as the inclusion map in $\mathcal{A}$ of the maximal $(C) *$-subalgebra of $\mathcal{A}$ on which $\alpha$ and $\beta$ agree.

For the classical theory, we will consider various categories of (pre)symplectic spaces. Let $\mathbb{K}$ be either $\mathbb{R}$ or $\mathbb{C}$. A pre-symplectic space over $\mathbb{K}$ is a pair $(V, \sigma)$ consisting of a $\mathbb{K}$-vector space $V$ equipped with an antisymmetric $\mathbb{K}$-bilinean form $\sigma: V \times V \rightarrow \mathbb{K}$. In the special case where, to each nonzero $u \in V$, there is a $v \in V$ with $\sigma(u, v) \neq 0$, we say that $(V, \sigma)$ is a weakly nondegenerate symplectic space. A symplectic map between two pre-symplectic spaces $(V, \sigma)$ and $\left(V^{\prime}, \sigma^{\prime}\right)$ is a $\mathbb{K}$-linear map $f: V \rightarrow V^{\prime}$ such that $\sigma^{\prime}(f u, f v)=\sigma(u, v)$ for all $u, v \in V$. We define a category preSymp $\mathbb{R}_{\mathbb{R}}$ to be the category of real pre-symplectic spaces, with injective symplectic maps as morphisms.

In the complex case, we wish to have available a complex conjugation as well. Thus preSympl $_{\mathbb{C}}$ will denote the category whose objects are triples $(V, \sigma, C)$, where $(V, \sigma)$ is a complex pre-symplectic space and $C: V \rightarrow V$ is an antilinear involution with $\sigma(\mathrm{Cu}, \mathrm{Cv})=$ $\overline{\sigma(u, v)}$, and with morphisms $(V, \sigma, C) \rightarrow\left(V^{\prime}, \sigma^{\prime}, C^{\prime}\right)$ given by an injective symplectic maps $f:(V, \sigma) \rightarrow\left(V^{\prime}, \sigma^{\prime}\right)$ such that $C^{\prime} \circ f=f \circ C$. We may regard preSympl $\mathbb{C}_{\mathbb{C}}$ as the category of complexified real pre-symplectic spaces. The full subcategory of preSympl $\left.\right|_{\mathbb{K}}$ whose objects are weakly nondegenerate will be denoted $\left.S_{y m p}\right|_{\mathbb{K}}$. (Note that if $(V, \sigma)$ is weakly nondegenerate then any symplectic map with domain $(V, \sigma)$ is injective.)

\footnotetext{
${ }^{4}$ Not sesquilinear, in the complex case.
} 
Given any $(V, \sigma) \in$ preSympl $_{\mathbb{R}}$ any vector subspace $W$ of $V$ induces $\left(W,\left.\sigma\right|_{W \times W}\right) \in$ preSympl $_{\mathbb{R}}$ and a canonical inclusion morphism $\left(W,\left.\sigma\right|_{W \times W}\right) \rightarrow(V, \sigma)$ in preSympl $\left.\right|_{\mathbb{R}}$. Similarly, given $(V, \sigma, C) \in$ preSympl $_{\mathbb{C}}$, any $C$-invariant subspace $W$ of $V$ induces analogous structures in preSympl $\left.\right|_{\mathbb{C}}$. (Weak nondegeneracy is not necessarily inherited under this restriction, which is why we work with pre-symplectic spaces.) The trivial vector space with zero symplectic form 5 (and trivial complex conjugation if $\mathbb{K}=\mathbb{C}$ ) provides an initial object in preSympl $_{\mathbb{K}}$. Intersections and unions are obtained in an obvious way from intersections and spans of ( $C$-invariant) vector subspaces; the equalizer of $f, g:(V, \sigma) \rightarrow\left(V^{\prime}, \sigma^{\prime}\right)$ in preSympl $_{\mathbb{R}}$ is the canonical inclusion morphism induced by the subspace $\operatorname{ker}(f-g)$ of $V$; in the complex case, with $f, g:(V, \sigma, C) \rightarrow\left(V^{\prime}, \sigma^{\prime}, C^{\prime}\right)$, the subspace $\operatorname{ker}(f-g)$ is $C$-invariant, and again defines the equalizer. Accordingly, preSympl $\mathbb{R}_{\mathbb{R}}$ and preSympl $_{\mathbb{C}}$ meet our general conditions to be categories of physical systems.

There is a useful functor $\mathscr{R}:$ preSympl $_{\mathbb{C}} \rightarrow \operatorname{preSympl}_{\mathbb{R}}:$ to each $(V, \sigma, C)$, it assigns $\left(V^{C},\left.\sigma\right|_{V^{C} \times V^{C}}\right)$, where $V^{C}:=\operatorname{ker}\left(C-\mathrm{id}_{V}\right)$, regarded as a real vector space; to any morphism $f:(V, \sigma, C) \rightarrow\left(V^{\prime}, \sigma^{\prime}, C^{\prime}\right)$, it assigns the restriction $\left.f\right|_{V^{C}}$, whose range is easily seen to lie in $W^{C^{\prime}}$. Moreover, this functor preserves weak nondegeneracy, intersections, unions and equalizers: for $C$-invariant subspaces $U, U^{\prime}$ of $V$, we have $\left(U \cap U^{\prime}\right)^{C}=U^{C} \cap U^{\prime C}$, $\left(U+U^{\prime}\right)^{C}=U^{C}+U^{\prime C}$, while for morphisms $f, g,(\operatorname{ker}(f-g))^{C}=\operatorname{ker}\left(\left.f\right|_{V^{C}}-\left.g\right|_{V^{C}}\right)$.

Locally covariant physical theories A locally covariant physical theory assigns physical systems to spacetimes and, importantly, to each hyperbolic embedding of spacetimes it assigns an embedding of the corresponding physical systems. It is represented by a (covariant) functor $\mathscr{A}:$ Loc $\rightarrow$ Phys (or from $\operatorname{Loc}_{0}$ if one restricts to connected spacetimes). The theories form the objects of a category LCT (or $L C T_{0}$ for theories on $\mathrm{Loc}_{0}$ ) in which morphisms between theories are natural transformations between the corresponding functors; this was used intensively in [27] but will not be needed here.

An important general feature of the BFV framework is that it contains a natural notion of dynamics: relative Cauchy evolution. Let $\boldsymbol{M}=(\mathcal{M}, \boldsymbol{g}, \mathfrak{o}, \mathfrak{t}) \in$ Loc be a globally hyperbolic spacetime. Given any symmetric $\boldsymbol{h} \in C_{0}^{\infty}\left(T_{2}^{0} \boldsymbol{M}\right)$ such that $\boldsymbol{g}+\boldsymbol{h}$ is a time-orientable Lorentz metric on $\mathcal{M}$, there is a unique choice of time-orientation $\mathfrak{t}_{\boldsymbol{h}}$ for $\boldsymbol{g}+\boldsymbol{h}$ that agrees with $\mathfrak{t}$ outside $K$. If $\boldsymbol{M}[\boldsymbol{h}]=\left(\mathcal{M}, \boldsymbol{g}+\boldsymbol{h}, \mathfrak{o}, \mathfrak{t}_{\boldsymbol{h}}\right)$ is a globally hyperbolic spacetime, we say that $\boldsymbol{h}$ is a globally hyperbolic perturbation of $\boldsymbol{M}$ and write $\boldsymbol{h} \in H(\boldsymbol{M})$. The subset of $\boldsymbol{h} \in H(\boldsymbol{M})$ with support in $K \subset \mathcal{M}$ is denoted $H(\boldsymbol{M} ; K)$. Clearly, $\boldsymbol{M}=\boldsymbol{M}[\mathbf{0}]$, where $\mathbf{0}$ is identically zero, and indeed $H(\boldsymbol{M})$ contains an open neighbourhood of $\mathbf{0}$ in the usual test-function topology on symmetric smooth compactly supported sections of $T_{2}^{0} \boldsymbol{M}$ (see $\S 7.1$ of [5]). We endow $H(\boldsymbol{M})$ with the subspace topology induced from $\mathscr{D}\left(T_{2}^{0} M\right)$.

For each $\boldsymbol{h} \in H(\boldsymbol{M})$, set $\mathcal{M}^{ \pm}=\mathcal{M} \backslash J_{\boldsymbol{M}}^{\mp}(\operatorname{supp} \boldsymbol{h})$, where $J_{\boldsymbol{M}}^{+/-}(S)$ denotes the causal future/past of a set $S$ (see [37] for definitions relating to causal structure). As shown in Sect. 3.4 of [27], the $\mathcal{M}^{ \pm}$are globally hyperbolic subsets of both $\boldsymbol{M}$ and $\boldsymbol{M}[\boldsymbol{h}]$ and therefore define canonical inclusions $\imath_{\boldsymbol{M}}^{ \pm}[\boldsymbol{h}]: \boldsymbol{M}^{ \pm}[\boldsymbol{h}] \rightarrow \boldsymbol{M}$ and $\jmath_{\boldsymbol{M}}^{ \pm}[\boldsymbol{h}] \rightarrow \boldsymbol{M}[\boldsymbol{h}]$, where $\boldsymbol{M}^{ \pm}[\boldsymbol{h}]:=\left.\boldsymbol{M}\right|_{\mathcal{M}^{ \pm}}$. Moreover, these canonical inclusions are Cauchy.

\footnotetext{
${ }^{5}$ This is non-degenerate, despite initial appearances.
} 
Then for any theory satisfying the time-slice property, which requires that the functor should map each Cauchy morphism to an isomorphism in Phys, we obtain isomorphisms

$$
\tau_{\boldsymbol{M}}^{ \pm}[\boldsymbol{h}]=\mathscr{A}\left(j_{\boldsymbol{M}}^{ \pm}[\boldsymbol{h}]\right) \circ\left(\mathscr{A}\left(\imath_{\boldsymbol{M}}^{ \pm}[\boldsymbol{h}]\right)\right)^{-1}: \mathscr{A}(\boldsymbol{M}) \rightarrow \mathscr{A}(\boldsymbol{M}[\boldsymbol{h}])
$$

and an automorphism $\operatorname{rce}_{\boldsymbol{M}}[\boldsymbol{h}]$ of $\mathscr{A}(\boldsymbol{M})$ given by

$$
\operatorname{rce}_{\boldsymbol{M}}[\boldsymbol{h}]=\left(\tau_{\boldsymbol{M}}^{-}[\boldsymbol{h}]\right)^{-1} \circ \tau_{\boldsymbol{M}}^{+}[\boldsymbol{h}],
$$

which is called the relative Cauchy evolution induced by $\boldsymbol{h}$. This formulation of the relative Cauchy evolution is equivalent to that given in BFV, by [27, Prop. 3.3].

Dynamical locality BFV emphasised that the standard structures of algebraic quantum field theory can be recovered from the locally covariant approach, on specialisation to particular spacetimes. The same can be done in the general case. Recalling that $\mathscr{O}(\boldsymbol{M})$ is the set of globally hyperbolic open subsets of $\boldsymbol{M}$ with at most finitely many mutually causally disjoint connected components, each nonempty $O \in \mathscr{O}(\boldsymbol{M})$ induces a canonical inclusion $\iota_{\boldsymbol{M} ; O}:\left.\boldsymbol{M}\right|_{O} \rightarrow \boldsymbol{M}$. Any locally covariant theory $\mathscr{A}:$ Loc $\rightarrow$ Phys, then assigns a physical system $\mathscr{A}\left(\left.\boldsymbol{M}\right|_{O}\right)$ and a morphism $\mathscr{A}\left(\iota_{\boldsymbol{M} ; O}\right): \mathscr{A}\left(\left.\boldsymbol{M}\right|_{O}\right) \rightarrow \mathscr{A}(\boldsymbol{M})$ embedding this as a subsystem of the physical system assigned to $\boldsymbol{M}$.

Accordingly, let Phys be any category obeying our minimal assumptions and let $\mathscr{A} \in$

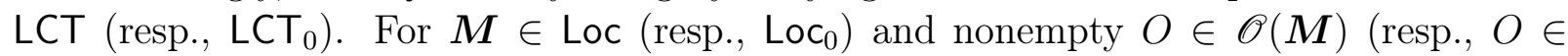
$\left.\mathscr{O}_{0}(\boldsymbol{M})\right)$, we define

$$
\mathscr{A}^{\mathrm{kin}}(\boldsymbol{M} ; O)=\mathscr{A}\left(\left.\boldsymbol{M}\right|_{O}\right), \quad \text { and } \quad \alpha_{\boldsymbol{M} ; O}^{\mathrm{kin}}=\mathscr{A}\left(\iota_{\boldsymbol{M} ; O}\right): \mathscr{A}^{\mathrm{kin}}(\boldsymbol{M} ; O) \rightarrow \mathscr{A}(\boldsymbol{M}) .
$$

We refer to the assignment $O \mapsto \alpha_{M ; O}^{\mathrm{kin}}$ as the kinematic net.

In general categories, it is better to focus on the morphism $\alpha_{\boldsymbol{M} ; O}^{\mathrm{kin}}:=\mathscr{A}\left(\iota_{\boldsymbol{M} ; O}\right)$, than its image in $\mathscr{A}(\boldsymbol{M})$, but for categories Phys such as those discussed in this paper, there is little harm in identifying $\mathscr{A}^{\text {kin }}(\boldsymbol{M} ; O)$ with this image.

One of the main ideas in [27] is that we may also use dynamics to identify local physics in theories obeying the time-slice property. This is done as follows. If $K$ is a compact subset of globally hyperbolic spacetime $\boldsymbol{M}$, any hyperbolic perturbation $\boldsymbol{h} \in H\left(\boldsymbol{M} ; K^{\perp}\right)$ represents a modification in the spacetime in regions causally inaccessible from $K$. We may test the sensitivity of subsystems of $\mathscr{A}(\boldsymbol{M})$ to these metric perturbations using the relative Cauchy evolution; in particular, we identify those subsystems that are insensitive to all such perturbations in $H\left(\boldsymbol{M} ; K^{\perp}\right)$ as candidates for being localised in $K$.

In the cases Phys $=\mathrm{Alg}, \mathrm{C}^{*}-\mathrm{Alg}$, this motivates the definition of a subalgebra

$$
\mathscr{A}^{\bullet}(\boldsymbol{M} ; K)=\left\{A \in \mathscr{A}(\boldsymbol{M}): \operatorname{rce}_{\boldsymbol{M}}[\boldsymbol{h}] A=A \text { for all } \boldsymbol{h} \in H\left(\boldsymbol{M} ; K^{\perp}\right)\right\}
$$

more generally, we may define a morphism $\alpha_{\boldsymbol{M} ; K}^{\bullet}$ as the unique (up to isomorphism) subobject of $\mathscr{A}(\boldsymbol{M})$ such that (i)

$$
\operatorname{rce}_{\boldsymbol{M}}[\boldsymbol{h}] \circ \alpha_{\boldsymbol{M} ; K}^{\bullet}=\alpha_{\boldsymbol{M} ; K}^{\bullet} \quad \forall \boldsymbol{h} \in H\left(\boldsymbol{M} ; K^{\perp}\right)
$$


and (ii) if any other morphism $\alpha$ satisfies Eq. (2.1) in place of $\alpha_{\boldsymbol{M} ; K}^{\bullet}$, then there is a unique morphism $\beta$ with $\alpha=\alpha_{\boldsymbol{M} ; K}^{\bullet} \circ \beta$. The existence of $\alpha_{\boldsymbol{M} ; K}^{\bullet}$ follows from the structural assumptions on Phys - indeed, we may write

$$
\alpha_{\boldsymbol{M} ; K}^{\bullet} \cong \bigwedge_{\boldsymbol{h} \in H\left(\boldsymbol{M} ; K^{\perp}\right)} \operatorname{eq}\left(\operatorname{rce}_{\boldsymbol{M}}[\boldsymbol{h}], \operatorname{id}_{\mathscr{A}(\boldsymbol{M})}\right)
$$

where $\bigwedge$ denotes the intersection in Phys. To obtain the local physics on a general $O \in$ $\mathscr{O}(\boldsymbol{M})$, we take the Phys-union over a suitable class of compact subsets of $O$,

$$
\alpha_{\boldsymbol{M} ; O}^{\mathrm{dyn}} \cong \bigvee_{K \in \mathscr{K}_{b}(\boldsymbol{M} ; O)} \alpha_{\boldsymbol{M} ; K}^{\bullet}
$$

Here $K \in \mathscr{K}_{b}(\boldsymbol{M} ; O)$ if is a finite union of causally disjoint subsets of $O$, each of which is the closure of a Cauchy ball $B$ with a relatively compact Cauchy development $D_{M}(B)$; a Cauchy ball $B$ is a subset of a Cauchy surface, for which there is a chart containing the closure of $B$, and in which $B$ is a nonempty open ball. (We also set $\mathscr{K}_{b}(\boldsymbol{M} ; \emptyset)=\{\emptyset\}$ by convention.) This differs slightly from the definition given first in [27], but is equivalent by Lemma 5.3 of that reference. As shown in [27], in spacetime dimension $n \geq 3$, each $K \in \mathscr{K}_{b}(\boldsymbol{M} ; O)$ has the property that $K^{\perp}$ has connected intersection with each connected component of $\boldsymbol{M}$; that this is not true in $n=2$ dimensions will have an interesting consequence in Sect. 4 .

Given these definitions, dynamical locality is defined as follows:

Definition 2.1 A theory $\mathscr{A} \in \mathrm{LCT}$ (resp., $\mathrm{LCT}_{0}$ ) obeys dynamical locality if it obeys the timeslice property and, additionally, for each $\boldsymbol{M} \in$ Loc (resp., Loc ${ }_{0}$ ) and all nonempty $O \in \mathscr{O}(\boldsymbol{M})$ (resp., $\left.\mathscr{O}_{0}(\boldsymbol{M})\right)$ we have $\mathscr{A}^{\mathrm{kin}}(\boldsymbol{M} ; O) \cong \mathscr{A}^{\mathrm{dyn}}(\boldsymbol{M} ; O)$, i.e., more abstractly, $\alpha_{\boldsymbol{M} ; O}^{\mathrm{kin}} \cong \alpha_{\boldsymbol{M} ; O}^{\mathrm{dyn}}$.

It is the main purpose of the present paper to investigate the extent to which this condition holds for the specific example of the minimally coupled Klein-Gordon field.

Before proceeding, we note one abstract result that will be useful to us. Suppose that Phys $_{1}$ and Phys ${ }_{2}$ are two categories of physical systems meeting the general criteria above, and let $\mathscr{F}:$ Phys $_{1} \rightarrow$ Phys $_{2}$ be a functor that preserves intersections, unions and equalizers, i.e.,

$\bigwedge_{i} \mathscr{F}\left(\alpha_{i}\right) \cong \mathscr{F}\left(\bigwedge_{i} \alpha_{i}\right), \quad \bigvee_{i} \mathscr{F}\left(\alpha_{i}\right) \cong \mathscr{F}\left(\bigvee_{i} \alpha_{i}\right), \quad \mathrm{eq}\left(\mathscr{F}\left(\alpha_{1}\right), \mathscr{F}\left(\alpha_{2}\right)\right) \cong \mathscr{F}\left(\mathrm{eq}\left(\alpha_{1}, \alpha_{2}\right)\right)$

for collections $\left(\alpha_{i}\right)$ of Phys $_{1}$-morphisms. Then any physical theory $\mathscr{A}_{1}:$ Loc $\rightarrow$ Phys $_{1}$ induces a theory $\mathscr{A}_{2}=\mathscr{F} \circ \mathscr{A}_{1}:$ Loc $\rightarrow$ Phys $_{2}$; moreover, if $\mathscr{A}_{1}$ is dynamically local, then so is $\mathscr{A}_{2}$. In particular, any dynamically local theory $\mathscr{L}:$ Loc $\rightarrow$ preSympl $_{\mathbb{C}}$ induces a dynamically local theory $\mathscr{R} \circ \mathscr{L}:$ Loc $\rightarrow$ preSympl $_{\mathbb{R}}$. 
The SPASs property The motivation underlying [27] is to understand the conditions under which a given theory may be regarded as displaying the same physics in all spacetimes. This is a difficult issue to make formal and it is conceivable that there could be a range of differing ways of doing so; however, we argued that (as well as local covariance) any such definition should have the following property: if two theories are given, each of which [under the given definition] individually represents the same physics in all spacetimes, and one theory is a subtheory of the other, and they coincide in one particular spacetime, then they coincide in all spacetimes. In the functorial context, this is made precise when one understands ' $\mathscr{A}$ is a subtheory of $\mathscr{B}$ ' to mean that there is a natural transformation $\eta: \mathscr{A} \rightarrow \mathscr{B}$, and ' $\mathscr{A}$ coincides with $\mathscr{B}$ in $\boldsymbol{M}$ ' to mean that the component $\eta_{M}$ is an isomorphism. We call this the SPASs property. Then the theories coincide in all spacetimes if and only if the functors $\mathscr{A}$ and $\mathscr{B}$ are naturally isomorphic, which is the usual understanding of functorial equivalence.

In 27] it was shown that the class of all locally covariant theories (even restricting to those with the time-slice property) is far too large to have the SPASs property. Thus there is more to the issue of SPASs than simple local covariance. However, the dynamically local theories do have the SPASs property (see Sect. 6.2 in [27]). While no claim is made that dynamical locality is the only possible definition that would qualify as a notion of SPASs, nor that the SPASs property is the only requirement one might reasonably demand of such a notion, it is currently the only contender. For this reason, and because dynamically local theories have a number of other nice properties, it is important to show that the main examples of quantum field theory in curved spacetime obey dynamical locality. This is the task to which we now turn.

\section{Classical theory of the Klein-Gordon field}

Functorial definition Given any $\boldsymbol{M} \in$ Loc, the minimally coupled Klein-Gordon equation is

$$
P_{M} \phi:=\left(\square_{M}+m^{2}\right) \phi=0,
$$

where $m \geq 0$ is constant. We write $\mathscr{L}_{\mathbb{K}}(\boldsymbol{M})$ to denote the space of smooth $\mathbb{K}$-valued solutions to this equation that have compact support on Cauchy surfaces in $\boldsymbol{M}$, and equip $\mathscr{L}_{\mathbb{K}}(\boldsymbol{M})$ with an antisymmetric bilinear form

$$
\sigma_{M}\left(\phi, \phi^{\prime}\right)=\int_{\Sigma}\left(\phi n^{a} \nabla_{a} \phi^{\prime}-\phi^{\prime} n^{a} \nabla_{a} \phi\right) d \Sigma,
$$

where $\Sigma$ is any Cauchy surface with future-pointing unit normal $n^{a}$; its values are independent of the choice of $\Sigma$. In the case where $\mathbb{K}=\mathbb{C}$, we use complex conjugation of functions as the antilinear involution on $\mathscr{L}_{\mathbb{C}}(\boldsymbol{M})$, i.e., $C_{\boldsymbol{M}} \phi=\bar{\phi}$. It is clear that $\left(\mathscr{L}_{\mathbb{R}}(\boldsymbol{M}), \sigma_{\boldsymbol{M}}\right) \in \operatorname{preSympl}_{\mathbb{R}}$ and $\left(\mathscr{L}_{\mathbb{C}}(\boldsymbol{M}), \sigma_{\boldsymbol{M}}, C_{\boldsymbol{M}}\right) \in$ preSympl $_{\mathbb{C}}$. We will focus on the complex case, from which we may read off all the structure of the real case by applying the functor $\mathscr{R}:$ preSympl $_{\mathbb{C}} \rightarrow$ preSympl $_{\mathbb{R}}$. To unburden the notation, we write $\mathscr{L}_{\mathbb{C}}$ as $\mathscr{L}$ for the rest of this section. 
A number of standard facts will be used in the sequel, and are collected here for reference. Let $E_{M}^{ \pm}: C_{0}^{\infty}(\boldsymbol{M}) \rightarrow C^{\infty}(\boldsymbol{M})$ be the advanced (-) and retarded (+) fundamental solutions, such that $\operatorname{supp} E_{M}^{ \pm} f \subset J_{M}^{ \pm}(\operatorname{supp} f)$. Then the advanced-minus-retarded fundamental solution is $E_{\boldsymbol{M}}=E_{\boldsymbol{M}}^{-}-E_{M}^{+}$, and acts as a bilinear form on $C_{0}^{\infty}(\boldsymbol{M})$ by

$$
E_{M}\left(f, f^{\prime}\right)=\left(E_{M} f^{\prime}\right)(f) \quad\left(f, f^{\prime} \in C_{0}^{\infty}(\boldsymbol{M})\right) .
$$

Then (see, e.g., [4 for proofs) $E_{M}$ has range and kernel

$$
\mathscr{L}(\boldsymbol{M})=E_{\boldsymbol{M}} C_{0}^{\infty}(\boldsymbol{M}), \quad \operatorname{ker} E_{\boldsymbol{M}}=P_{\boldsymbol{M}} C_{0}^{\infty}(\boldsymbol{M})
$$

and there is an identity

$$
\sigma_{M}\left(E_{M} f, \phi\right)=\int_{M} \phi(p) f(p) d \operatorname{vol}_{M}(p)
$$

for $f \in C_{0}^{\infty}(\boldsymbol{M}), \phi \in \mathscr{L}(\boldsymbol{M})$, which implies

$$
\sigma_{M}\left(E_{M} f, E_{M} f^{\prime}\right)=E_{M}\left(f, f^{\prime}\right)
$$

and also shows that $\sigma_{M}$ is weakly nondegenerate.

Now suppose a morphism $\psi: \boldsymbol{M} \rightarrow \boldsymbol{N}$ is given, and define the push-forward on test functions by $\psi_{*} f$

$$
\left(\psi_{*} f\right)(p)= \begin{cases}f\left(\psi^{-1}(p)\right) & p \in \psi(\boldsymbol{M}) \\ 0 & \text { otherwise }\end{cases}
$$

for $f \in C_{0}^{\infty}(\boldsymbol{M})$. As $\mathscr{L}(\boldsymbol{M})=E_{\boldsymbol{M}} C_{0}^{\infty}(\boldsymbol{M})$ and

$$
\psi_{*} \operatorname{ker} E_{\boldsymbol{M}}=\psi_{*} P_{\boldsymbol{M}} C_{0}^{\infty}(\boldsymbol{M})=P_{\boldsymbol{N}} \psi_{*} C_{0}^{\infty}(\boldsymbol{M}) \subset \operatorname{ker} E_{\boldsymbol{N}},
$$

there is a unique linear map $\mathscr{L}_{\mathbb{K}}(\psi): \mathscr{L}(\boldsymbol{M}) \rightarrow \mathscr{L}(\boldsymbol{N})$, with $\mathscr{L}(\psi) \circ E_{\boldsymbol{M}}=E_{\boldsymbol{N}} \circ \psi_{*}$ which extends a solution on $\boldsymbol{M}$ to one on $\boldsymbol{N}$. Covariance of the field equation together with uniqueness of advanced/retarded solutions to the inhomogeneous Klein-Gordon equation gives the identity $\psi^{*} E_{\boldsymbol{N}} \psi_{*}=E_{\boldsymbol{M}}$ on $C_{0}^{\infty}(\boldsymbol{M})$ and it follows that $\psi^{*} \mathscr{L}(\psi) \phi=\phi$ for all $\phi \in \mathscr{L}(\boldsymbol{M})$, so $\mathscr{L}(\psi)$ is injective.

Owing to the calculation

$$
\begin{aligned}
\sigma_{\boldsymbol{N}}\left(\mathscr{L}(\psi) E_{\boldsymbol{M}} f, \mathscr{L}(\psi) E_{\boldsymbol{M}} f^{\prime}\right) & =\sigma_{\boldsymbol{N}}\left(E_{\boldsymbol{N}} \psi_{*} f, E_{\boldsymbol{N}} \psi_{*} f^{\prime}\right)=E_{\boldsymbol{N}}\left(\psi_{*} f, \psi_{*} f^{\prime}\right)=E_{\boldsymbol{M}}\left(f, f^{\prime}\right) \\
& =\sigma_{\boldsymbol{M}}\left(E_{\boldsymbol{M}} f, E_{\boldsymbol{M}} f^{\prime}\right)
\end{aligned}
$$

and the obvious properties in relation to compositions and identities, $\mathscr{L}$ is a functor from Loc to preSympl $;$; as $\mathscr{L}(\boldsymbol{M})$ is weakly nondegenerate for every $\boldsymbol{M}$, we describe $\mathscr{L}$ as weakly nondegenerate. Accordingly, there is a unique factorization of $\mathscr{L}$ through the forgetful functor from Sympl $\mathbb{C}_{\mathbb{C}}$ to preSympl $_{\mathbb{C}}$. 
Time-slice property and relative Cauchy evolution The following result is a mild extension of a standard argument [19, Lem. A.3].

Lemma 3.1 (i) Let $K$ be a compact subset of $\boldsymbol{M}$, with $K \subset O \in \mathscr{O}(\boldsymbol{M})$. Then there exists a smooth function $\chi$ such that every $\phi \in \mathscr{L}(\boldsymbol{M})$ with $\operatorname{supp} \phi \subset J_{\boldsymbol{M}}(K)$ may be written as $\phi=E_{\boldsymbol{M}} P_{\boldsymbol{M}} \chi \phi$ with $P_{\boldsymbol{M}} \chi \phi \in C_{0}^{\infty}(O)$. (ii) If $O \in \mathscr{O}(\boldsymbol{M})$ contains a Cauchy surface of $\boldsymbol{M}$, then there exists a smooth function $\chi$ such that every $\phi \in \mathscr{L}(\boldsymbol{M})$ may be written as $\phi=E_{\boldsymbol{M}} P_{\boldsymbol{M}} \chi \phi$ with $P_{\boldsymbol{M}} \chi \phi \in C_{0}^{\infty}(O)$.

Proof: (i) As $K$ is compact and contained in $O, J_{M}(K)$ has compact intersection with Cauchy surfaces of $O$ (using e.g., [4, Cor. A.5.4] and causal convexity of $O$ ). Choosing Cauchy surfaces $\Sigma^{ \pm}$of $O$ passing to the future $(+)$ and past $(-)$ of $K$, the sets $K^{ \pm}=$ $J_{\boldsymbol{M}}(K) \cap \Sigma^{ \pm}$are compact and give a cover

$$
J_{M}(K)=J_{M}^{+}\left(K^{+}\right) \cup J_{M}^{-}\left(K^{-}\right) \cup K_{0}
$$

in which the first two sets on the right-hand side are closed and disjoint, while $K_{0}=$ $J_{\boldsymbol{M}}(K) \cap\left(J_{\boldsymbol{M}}^{-}\left(K^{+}\right) \cap J_{\boldsymbol{M}}^{+}\left(K^{-}\right)\right)$is compact (see e.g., [4, Lem. A.5.7]) and contained in $O$. We may therefore choose $\chi \in C^{\infty}(\boldsymbol{M})$ with $\chi=0$ on $J_{M}^{+}\left(K^{+}\right)$and $\chi=1$ on $J_{M}^{-}\left(K^{-}\right)$ (see, e.g., [1, Prop. 5.5.8]). Following a standard argument [19], $P_{\boldsymbol{M}} \chi \phi$ is supported in the compact set $K_{0} \subset O$; on support grounds, it follows that

$$
\chi \phi=E_{M}^{-} P_{M} \chi \phi, \quad(\chi-1) \phi=E_{M}^{+} P_{M} \chi \phi
$$

and hence $\phi=E_{\boldsymbol{M}} P_{\boldsymbol{M}} \chi \phi \in E_{\boldsymbol{M}} C_{0}^{\infty}(O)$.

(ii) If $O \in \mathscr{O}(\boldsymbol{M})$ contains a Cauchy surface $\Sigma$ of $\boldsymbol{M}$, then it also contains Cauchy surfaces $\Sigma^{ \pm}$passing to the future/past of $\Sigma$. As $J_{M}^{+}\left(\Sigma^{+}\right)$and $J_{M}^{-}\left(\Sigma^{-}\right)$are closed and disjoint, we may choose $\chi \in C^{\infty}(\boldsymbol{M})$ with $\chi=0$ on $J_{\boldsymbol{M}}^{+}\left(\Sigma^{+}\right)$and $\chi=1$ on $J_{\boldsymbol{M}}^{-}\left(\Sigma^{-}\right)$; the argument proceeds as before.

It follows immediately that $\mathscr{L}$ has the time-slice property: if $\psi: \boldsymbol{L} \rightarrow \boldsymbol{M}$ is Cauchy, then $\mathscr{L}(\psi)$ is surjective in addition to being symplectic and injective and therefore has a symplectic inverse. Moreover, because $\psi(\boldsymbol{L})$ contains a Cauchy surface of $\boldsymbol{M}$ we may also characterize $\mathscr{L}(\psi) \phi$ as the unique $P_{\boldsymbol{M}}$-solution on $\boldsymbol{M}$ whose pull-back to $\boldsymbol{L}$ coincides with $\phi$. This allows us to read off the relative Cauchy evolution. Given $\boldsymbol{h} \in H(\boldsymbol{M})$ and setting $\mathcal{M}^{ \pm}=\boldsymbol{M} \backslash J_{M}^{\mp}(\operatorname{supp} \boldsymbol{h})$,

$$
\operatorname{rce}_{\boldsymbol{M}}[\boldsymbol{h}] \phi=\mathscr{L}\left(\imath_{\boldsymbol{M}}^{-}[\boldsymbol{h}]\right) \circ \mathscr{L}\left(\jmath_{\boldsymbol{M}}^{-}[\boldsymbol{h}]\right)^{-1} \circ \mathscr{L}\left(\jmath_{\boldsymbol{M}}^{+}[\boldsymbol{h}]\right) \circ \mathscr{L}\left(\imath_{\boldsymbol{M}}^{+}[\boldsymbol{h}]\right)^{-1} \phi \in \mathscr{L}(\boldsymbol{M})
$$

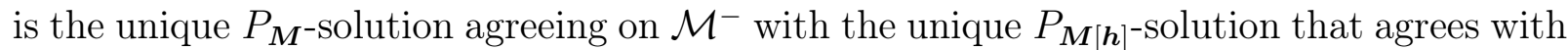
$\phi$ on $\mathcal{M}^{+}$. Explicit formulae are given in Sec. 4 of BFV (notation differs) and Appendix B.

An important example arises in the massless case. If $\boldsymbol{M}$ has one or more components with compact Cauchy surfaces, there are nontrivial solutions $\phi$ which are locally constant, i.e., take constant (possibly different) values on each connected component. These are solutions to the massless Klein-Gordon equation for any smooth metric on the underlying manifold of $\boldsymbol{M}$ and are therefore fixed points under arbitrary relative Cauchy evolution. 
As first pointed out by BFV, the functional derivative of the relative Cauchy evolution with respect to the metric is closely related to the stress energy tensor. In the present setting this can be seen as follows. Let $\operatorname{Sym}(\boldsymbol{M})$ denote the space of smooth symmetric second rank covariant tensor fields of compact support on $\boldsymbol{M}$, and $\operatorname{Sym}(\boldsymbol{M} ; O)$ the subspace consisting of those supported in $O \subset \boldsymbol{M}$. For $\boldsymbol{f} \in \operatorname{Sym}(\boldsymbol{M}), s \mapsto \operatorname{rce}_{\boldsymbol{M}}[s \boldsymbol{f}]$ is differentiable at $s=0$ in the weak symplectic topology, i.e., there exists a linear map $F_{\boldsymbol{M}}[\boldsymbol{f}]: \mathscr{L}(\boldsymbol{M}) \rightarrow$ $\mathscr{L}(\boldsymbol{M})$ such that

$$
\sigma_{M}\left(F_{\boldsymbol{M}}[\boldsymbol{f}] \phi, \phi^{\prime}\right)=\left.\frac{d}{d s} \sigma_{\boldsymbol{M}}\left(\operatorname{rce}_{\boldsymbol{M}}[s \boldsymbol{f}] \phi, \phi^{\prime}\right)\right|_{s=0} \quad(\phi \in \mathscr{L}(\boldsymbol{M}))
$$

for any $\boldsymbol{f} \in \operatorname{Sym}(\boldsymbol{M})$. The maps $F_{\boldsymbol{M}}[\boldsymbol{f}]$ are given in BFV 6

$$
\begin{aligned}
F_{M}[\boldsymbol{f}] \phi & =E_{M}\left(\frac{1}{2}\left(\nabla^{a} f_{b}^{b}\right) \nabla_{a} \phi-\nabla_{a} f^{a b} \nabla_{b} \phi\right) \\
& =E_{M}\left(\frac{1}{2} \nabla^{a} f_{b}^{b} \nabla_{a} \phi-\nabla_{a} f^{a b} \nabla_{b} \phi+\frac{1}{2} m^{2} \phi f^{b}{ }_{b}\right)
\end{aligned}
$$

where the Klein-Gordon equation was employed in the last step. However we note that a more informative form can be given: it turns out that

$$
\sigma_{\boldsymbol{M}}\left(F_{\boldsymbol{M}}(\boldsymbol{f}) \phi, \bar{\phi}\right)=\int_{\boldsymbol{M}} f_{a b} T_{\boldsymbol{M}}^{a b}[\phi] d \mathrm{vol}_{\boldsymbol{M}},
$$

where $\boldsymbol{T}_{\boldsymbol{M}}[\phi]$ is the classical stress-energy tensor on $\boldsymbol{M}$ for the solution $\phi$ :

$$
T_{M}^{a b}[\phi]=\left(\nabla^{(a} \bar{\phi}\right)\left(\nabla^{b)} \phi\right)-\frac{1}{2} g^{a b} g^{c d}\left(\nabla_{c} \bar{\phi}\right)\left(\nabla_{d} \phi\right)+\frac{1}{2} m^{2}|\phi|^{2} g^{a b} .
$$

To see this, we use (3.3) to note that

$$
\sigma_{M}\left(F_{M}(\boldsymbol{f}) \phi, \bar{\phi}\right)=\int_{M} \bar{\phi}\left(\frac{1}{2} \nabla^{a} f^{b}{ }_{b} \nabla_{a} \phi-\nabla_{a} f^{a b} \nabla_{b} \phi+\frac{1}{2} m^{2} \phi f^{b}{ }_{b}\right) d \operatorname{vol}_{M}
$$

and then integrate by parts in the first two terms, using the fact that $f$ is compactly supported and symmetric to discard boundary terms and thereby obtain the required result.

Let us also recall that if $u$ is a timelike unit vector then

$$
T_{M}^{a b}[\phi] u_{a} u_{b}=\frac{1}{2} h^{a b} \nabla_{a} \bar{\phi} \nabla_{b} \phi+\frac{1}{2} m^{2}|\phi|^{2},
$$

where $h^{a b}=2 u^{a} u^{b}-g^{a b}$ is positive definite. Accordingly, vanishing of $\boldsymbol{T}_{\boldsymbol{M}}[\phi]$ at a point $p$ implies that $\nabla \phi$ vanishes there; for $m>0$ we may also conclude that $\phi$ also vanishes at $p$.

${ }^{6}$ Although BFV give a formula for the derivative, the precise sense in which differentiability is understood was not delineated there, nor was differentiability actually proved. This is remedied here; see Appendix B for a proof of differentiability. The weak symplectic topology plays a role in general investigations of the canonical commutation relations [36] and appears the natural choice here. 
Dynamical locality An immediate consequence of the definition is that the kinematic subspaces are given, for nonempty $O \in \mathscr{O}(\boldsymbol{M})$, by

$$
\mathscr{L}^{\text {kin }}(\boldsymbol{M} ; O)=\operatorname{Ran}\left(\mathscr{L}\left(\iota_{\boldsymbol{M} ; O}\right)\right)=E_{\boldsymbol{M}} C_{0}^{\infty}(O) .
$$

As a slight digression, which will be useful later, we note that if $O, O^{\prime} \in \mathscr{O}(\boldsymbol{M})$ are nonempty and causally disjoint, then

$$
\sigma_{\boldsymbol{M}}\left(\mathscr{L}^{\mathrm{kin}}(\boldsymbol{M} ; O), \mathscr{L}^{\mathrm{kin}}\left(\boldsymbol{M} ; O^{\prime}\right)\right)=\{0\}
$$

because $E_{M}$ vanishes on pairs of test functions with causally disjoint supports. By analogy with the situation in algebraic quantum field theory, we call this Einstein causality. It follows that we also have the analogue of the extended locality property of QFT [41, 34,

$$
\mathscr{L}^{\text {kin }}\left(\boldsymbol{M} ; O_{1}\right) \cap \mathscr{L}^{\mathrm{kin}}\left(\boldsymbol{M} ; O_{2}\right)=\{0\} .
$$

For suppose $\phi \neq 0$ is an element of the intersection, then it can be written as $\phi=$ $\mathscr{L}\left(\iota_{\boldsymbol{M} ; O}\right) \hat{\phi}$ for some $\hat{\phi} \in \mathscr{L}\left(\left.\boldsymbol{M}\right|_{O}\right)$. By weak nondegeneracy, there exists $\hat{\phi}^{\prime} \in \mathscr{L}\left(\left.\boldsymbol{M}\right|_{O}\right)$ with $\sigma_{\boldsymbol{M} \mid O}\left(\hat{\phi}, \hat{\phi}^{\prime}\right) \neq 0$. But then also $\sigma_{\boldsymbol{M}}\left(\phi, \mathscr{L}\left(\iota_{\boldsymbol{M} ; O}\right) \hat{\phi}^{\prime}\right) \neq 0$, which is a contradiction because $\phi$ may also be regarded as an element of $\mathscr{L}^{\text {kin }}\left(\boldsymbol{M} ; O^{\prime}\right)$.

We now proceed to compute the dynamical subspaces.

Proposition 3.2 Let $K$ be any compact subset of $\boldsymbol{M} \in$ Loc. Then

$$
\mathscr{L}^{\bullet}(\boldsymbol{M} ; K)=\left\{\phi \in \mathscr{L}(\boldsymbol{M}): \operatorname{supp} \boldsymbol{T}_{\boldsymbol{M}}[\phi] \subset J_{\boldsymbol{M}}(K)\right\} .
$$

Proof: Suppose that $\phi \in \mathscr{L}^{\bullet}(\boldsymbol{M} ; K)$. Given any $\boldsymbol{f} \in \operatorname{Sym}\left(\boldsymbol{M} ; K^{\perp}\right)$, there is an interval containing $s=0$ for which $s \boldsymbol{f} \in H\left(\boldsymbol{M} ; K^{\perp}\right)$; as $\operatorname{rce}_{\boldsymbol{M}}[s \boldsymbol{f}] \phi=\phi$ for all such $s$ we may differentiate to obtain $F_{\boldsymbol{M}}[\boldsymbol{f}] \phi=0$ for all such $\boldsymbol{f}$. It follows immediately from (3.7) that $\boldsymbol{T}_{\boldsymbol{M}}[\phi]$ is supported in $J_{\boldsymbol{M}}(K)$.

Conversely, suppose $\phi \in \boldsymbol{T}_{\boldsymbol{M}}[\phi]$ vanishes in $K^{\perp}$. Then $\nabla \phi$ vanishes in $K^{\perp}$ and so $\phi$ is constant in each connected component of $K^{\perp}$. Accordingly, $\phi$ is also a Klein-Gordon solution with respect to any perturbed metric induced by $\boldsymbol{h} \in H\left(\boldsymbol{M} ; K^{\perp}\right)$, which shows that $\operatorname{rce}_{\boldsymbol{M}}[\boldsymbol{h}] \phi=\phi$ for all such $\boldsymbol{h}$, i.e., $\phi \in \mathscr{L}^{\bullet}(\boldsymbol{M} ; K)$.

Note that $\mathscr{L} \bullet(M ; K)$ includes solutions whose support extends to the boundary of $J_{M}(K)$. By contrast, if $O$ is a nonempty open relatively compact globally hyperbolic subset of $\boldsymbol{M}$, solutions in $\mathscr{L}^{\mathrm{kin}}(\boldsymbol{M} ; O)$ have (closed) support contained in $J_{\boldsymbol{M}}(O)$ which is a proper subset of $J_{\boldsymbol{M}}(\mathrm{cl}(O))$. Thus we see that $\mathscr{L}^{\mathrm{kin}}(\boldsymbol{M} ; O)$ is a proper subset of $\mathscr{L} \bullet(M ; \operatorname{cl}(O))$ in this case. In general, we also have

Lemma $3.3 \mathscr{L}^{\mathrm{kin}}(\boldsymbol{M} ; O) \subset \mathscr{L}^{\mathrm{dyn}}(\boldsymbol{M} ; O)$ for all nonempty $O \in \mathscr{O}(\boldsymbol{M})$.

Proof: Suppose $\phi=\mathscr{L}^{\mathrm{kin}}(\boldsymbol{M} ; O)$, so $\phi=E_{\boldsymbol{M}} f$ for some $f \in C_{0}^{\infty}(O)$. We may decompose $f$ as a finite sum $f=\sum f_{i}$ in which $\operatorname{supp} f_{i} \in \mathscr{K}(\boldsymbol{M} ; O)$. (Take an open cover of supp $f$ by diamonds and pass to a finite subcover and then a subordinate partition of unity.) Each 
$E_{\boldsymbol{M}} f_{i}$ has support in $J_{\boldsymbol{M}}\left(\operatorname{supp} f_{i}\right)$ and hence belongs to $\mathscr{L}^{\bullet}\left(\boldsymbol{M} ; \operatorname{supp} f_{i}\right)$, which shows that $\phi=\sum_{i} E_{\boldsymbol{M}} f_{i} \in \mathscr{L}^{\mathrm{dyn}}(\boldsymbol{M} ; O)$.

At this stage, the mass parameter $m$ becomes important. If $m>0$ then, using Prop. 3.2 and Lem. 3.1(i), we have

$$
\mathscr{L}^{\bullet}(\boldsymbol{M} ; K)=\left\{\phi \in \mathscr{L}(\boldsymbol{M}): \operatorname{supp} \phi \subset J_{\boldsymbol{M}}(K)\right\} \subset E_{\boldsymbol{M}} C_{0}^{\infty}(O)=\mathscr{L}^{\mathrm{kin}}(\boldsymbol{M} ; O)
$$

for every $K \in \mathscr{K}(\boldsymbol{M} ; O)$ and $O \in \mathscr{O}(\boldsymbol{M})$. Taking a union over all such $K$, we obtain the reverse inclusion to Lem. 3.3. As dynamical locality of $\mathscr{L}=\mathscr{L}_{\mathbb{C}}$ implies that of $\mathscr{L}_{\mathbb{R}}$, we have proved:

Theorem 3.4 The classical Klein-Gordon theory $\mathscr{L}_{\mathbb{K}}$ is dynamically local in LCT (and hence its restriction to $\mathrm{Loc}_{0}$ is dynamically local in $\mathrm{LCT}_{0}$ ) for all $m>0$.

Now consider the case $m=0$. Any function on $\boldsymbol{M}$ that is locally constant (i.e., constant on each connected component of $\boldsymbol{M}$ ) satisfies the field equation; we denote by $\mathscr{L}_{\text {l.c. }}(\boldsymbol{M})$ the space of locally constant solutions with compact support on Cauchy surfaces, which has dimension equal to the number of compact connected components of $\boldsymbol{M}$. (In the case where $\boldsymbol{M}$ has purely noncompact Cauchy surfaces $\mathscr{L}_{\text {l.c. }}(\boldsymbol{M})$ is trivial.) Now from Prop. 3.2, we know that

$$
\mathscr{L} \bullet(M ; K)=\left\{\phi \in \mathscr{L}(\boldsymbol{M}): \operatorname{supp} \nabla \phi \subset J_{\boldsymbol{M}}(K)\right\}
$$

for any compact set $K$, so any $\phi \in \mathscr{L}^{\bullet}(\boldsymbol{M} ; K)$ is constant on each connected component of $K^{\perp}$. This allows us to prove:

Lemma 3.5 If $K \subset \boldsymbol{M}$ is compact, and $K^{\perp}$ has connected intersection with each connected component of $\boldsymbol{M}$, then

$$
\mathscr{L} \bullet(M ; K)=\left\{\phi \in \mathscr{L}(\boldsymbol{M}): \operatorname{supp} \phi \subset J_{\boldsymbol{M}}(K)\right\}+\mathscr{L}_{\text {l.c. }}(\boldsymbol{M}) .
$$

Proof: The remarks above and the hypothesis on $K$ permit us to write any $\phi \in \mathscr{L}^{\bullet}(\boldsymbol{M} ; K)$ as a sum $\phi=\phi_{\text {l.c. }}+\phi_{0}$, where $\phi_{\text {l.c. }} \in \mathscr{L}_{\text {l.c. }}(\boldsymbol{M})$ is locally constant and $\phi_{0}$ is supported in $J_{M}(K)$. This gives the inclusion of the left-hand side in the right; for the reverse inclusion, we use the fact that every element of $\mathscr{L}_{\text {l.c. }}(\boldsymbol{M})$ is invariant under arbitrary classical relative Cauchy evolution.

If $O \in \mathscr{O}(\boldsymbol{M})$ is nonempty, $\mathscr{L}^{\mathrm{dyn}}(\boldsymbol{M} ; O)$ is the span of the subspaces $\mathscr{L} \bullet(\boldsymbol{M} ; K)$ for $K \in \mathscr{K}_{b}(\boldsymbol{M} ; O)$, defined before Def. 2.1. In spacetime dimension $n>2$ each such $K$ meets the hypotheses of Lem. 3.5 and we easily see that

$$
\mathscr{L}^{\mathrm{dyn}}(\boldsymbol{M} ; O)=\mathscr{L}^{\mathrm{kin}}(\boldsymbol{M} ; O)+\mathscr{L}_{\text {l.c. }}(\boldsymbol{M}) .
$$

Spacetime dimension $n=2$ is complicated by the fact that every nonempty $K \in \mathscr{K}_{b}(\boldsymbol{M} ; O)$ has disconnected intersection with at least one of the connected components of $\boldsymbol{M}$. This is worthy of more discussion, but for the present, we simply observe that $\mathscr{L}^{\mathrm{dyn}}(\boldsymbol{M} ; O)$ contains $\mathscr{L}^{\text {kin }}(\boldsymbol{M} ; O)+\mathscr{L}_{\text {l.c. }}(\boldsymbol{M})$.

Summarising, we have shown: 
Theorem 3.6 The classical Klein-Gordon theory $\mathscr{L}_{\mathbb{K}}$ is not dynamically local for $m=0$.

The classical massless Klein-Gordon field is singled out by not obeying the dynamical locality property. As we will see below, this also propagates to the quantum field theory. Although the discrepancy between the kinematic and dynamical subspaces is slight and under full control, we adopt the viewpoint that the failure of dynamical locality should be taken seriously as an indication of a defect in the usual treatment of the massless minimally coupled model. The root cause is easily seen: namely, the rigid gauge symmetry $\phi \mapsto \phi+$ const in the Lagrangian. In the next section, we show how the massless theory can be formulated in a dynamically local way, by treating it as a (rather simple) gauge theory.

To conclude this section, we summarise a number of features of the Klein-Gordon theory with arbitrary mass $m \geq 0$ from the above discussion. For $\mathscr{L}=\mathscr{L}_{\mathbb{C}}$ or $\mathscr{L}_{\mathbb{R}}$ we have:

$(\mathscr{L} 1) \mathscr{L}$ has a smooth stress-energy tensor, i.e., the relative Cauchy evolution is differentiable in the weak symplectic topology as in (3.5), and the resulting maps $F_{M}[\boldsymbol{f}]$ obey

$$
\sigma_{\boldsymbol{M}}\left(F_{\boldsymbol{M}}[\boldsymbol{f}] \phi, C_{\boldsymbol{M}} \phi\right)=\int f_{a b} T_{M}^{a b}[\phi] d \mathrm{vol} \quad(\boldsymbol{f} \in \operatorname{Sym}(\boldsymbol{M} ; O)),
$$

where $\boldsymbol{T}_{\boldsymbol{M}}[\phi] \in C^{\infty}\left(T_{0}^{2}(\boldsymbol{M})\right)$ is a smooth conserved symmetric tensor field for each $\phi \in \mathscr{L}(\boldsymbol{M})$ (in the case $\mathbb{K}=\mathbb{R}$, take $C_{\boldsymbol{M}}$ to be the identity).

$(\mathscr{L} 2)$ For each $O \in \mathscr{O}(\boldsymbol{M})$ containing supp $\boldsymbol{f}$, we have im $F_{\boldsymbol{M}}[\boldsymbol{f}] \subset \mathscr{L}^{\text {kin }}(\boldsymbol{M} ; O)$.

$(\mathscr{L} 3) \mathscr{L}$ obeys extended locality.

$(\mathscr{L} 4)$ The stress-energy tensor is sufficient to define the dynamical subspaces, i.e.,

$$
\mathscr{L} \bullet(\boldsymbol{M} ; K)=\bigcap_{\boldsymbol{f} \in \operatorname{Sym}\left(\boldsymbol{M} ; K^{\perp}\right)} \operatorname{ker} F_{\boldsymbol{M}}[\boldsymbol{f}] .
$$

One might expect these properties to hold for wide range of theories of interest. Later, it will be useful to have the following technical result, in which dynamical locality is not assumed.

Proposition 3.7 Suppose $\mathscr{L}:$ Loc $\rightarrow$ preSympl $_{\mathbb{K}}$ is weakly nondegenerate and obeys

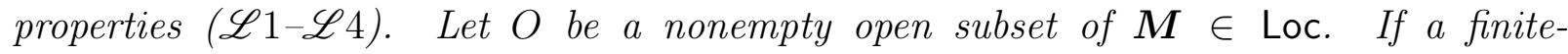
dimensional subspace $Y \subset \mathscr{L}(\boldsymbol{M})$ is invariant under $F_{\boldsymbol{M}}[\boldsymbol{f}]$ for every $\boldsymbol{f} \in \operatorname{Sym}(\boldsymbol{M} ; O)$ then $Y \subset \bigcap_{\boldsymbol{f} \in \operatorname{Sym}(\boldsymbol{M} ; O)} \operatorname{ker} F_{\boldsymbol{M}}[\boldsymbol{f}]$. (In particular, every $\phi \in Y$ has vanishing stress-energy tensor in $O$.) In the case $O=K^{\perp}$ for compact $K$, this implies that $Y \subset \mathscr{L} \bullet(M ; K)$.

Remark: In particular, we any finite dimensional subspace $Y$ that is invariant under rce $_{\boldsymbol{M}}[\boldsymbol{h}]$ for all $\boldsymbol{h} \in H\left(\boldsymbol{M} ; K^{\perp}\right)$ obeys $Y \subset \mathscr{L}^{\bullet}(\boldsymbol{M} ; K)$. The result also holds with Loc replaced by Loc $_{0}$ throughout. 
Proof. Let $p \in O$ be arbitrary. In any neighbourhood $\tilde{O}$ of $p$ with $\tilde{O} \subset O$ choose causally disjoint open subsets $O_{1}, \ldots, O_{1+\operatorname{dim} Y}$ and consider the subspaces

$$
Y_{i}=\left.\bigvee_{\boldsymbol{f} \in \operatorname{Sym}\left(\boldsymbol{M} ; O_{i}\right)} \operatorname{im} F_{\boldsymbol{M}}[\boldsymbol{f}]\right|_{Y}
$$

of $Y$ (where $\bigvee$ denotes the span of subspaces). Using $(\mathscr{L} 2)$ and $(\mathscr{L} 3)$, it is clear that the $Y_{i}$ constitute $1+\operatorname{dim} Y$ subspaces of $Y$ with trivial pairwise intersections,so at least one of the $Y_{i}$, say $Y_{1}$, must be trivial. For each $\phi \in Y$ we must therefore have $F_{\boldsymbol{M}}[\boldsymbol{f}] \phi=0$ for every $\boldsymbol{f} \in \operatorname{Sym}\left(\boldsymbol{M} ; O_{1}\right)$.

The existence of a smooth stress-energy tensor entails, by a polarisation argument, that for each $\phi^{\prime} \in \mathscr{L}(\boldsymbol{M})$ there is a smooth tensor field $\boldsymbol{T}_{\boldsymbol{M}}\left[\phi, \phi^{\prime}\right]$ with $\sigma_{\boldsymbol{M}}\left(F_{\boldsymbol{M}}[\boldsymbol{f}] \phi, C_{\boldsymbol{M}} \phi^{\prime}\right)=$ $\int_{\boldsymbol{M}} T_{\boldsymbol{M}}^{a b}\left[\phi, \phi^{\prime}\right] f_{a b} d \mathrm{vol}$, where $C_{\boldsymbol{M}}$ is the conjugation in $\mathscr{L}(\boldsymbol{M})$. The above remarks show that $\boldsymbol{T}_{\boldsymbol{M}}\left[\phi, \phi^{\prime}\right]$ vanishes identically in $O_{1} \subset \tilde{O}$. As $\tilde{O}$ was arbitrary, it follows by continuity that $\boldsymbol{T}_{\boldsymbol{M}}\left[\phi, \phi^{\prime}\right]$ vanishes at $p$, which was an arbitrary point of $O$. Consequently, $\sigma_{\boldsymbol{M}}\left(F_{\boldsymbol{M}}[\boldsymbol{f}] \phi, C_{\boldsymbol{M}} \phi^{\prime}\right)=0$ for arbitrary $\phi^{\prime} \in \mathscr{L}(\boldsymbol{M}), \boldsymbol{f} \in \mathscr{L}(\boldsymbol{M} ; O)$. As $\sigma_{\boldsymbol{M}}$ is weakly nondegenerate, we conclude that $F_{\boldsymbol{M}}[\boldsymbol{f}] \phi=0$ for all $\phi \in Y$ and $\boldsymbol{f} \in \operatorname{Sym}(\boldsymbol{M} ; O)$. The last statement follows from $(\mathscr{L} 4)$.

\section{The massless current}

Our eventual aim is to quantize the massless minimally coupled model as a gauge theory, following the general lines of treatments of the electromagnetic field [20, 25] or its analogues [38] (although we will not make the cohomological restrictions imposed in these references). In this section, we describe the underlying classical field theory, aiming for a dynamically local theory valued in preSympl $\mathbb{K}_{\mathbb{K}}$. As before, we will focus on the complex case, dropping $\mathbb{C}$ from the notation, with $\mathscr{L}$ denoting the massless Klein-Gordon field as formulated above (in preSympl $\mathbb{C}_{\mathbb{C}}$.

It is convenient to employ differential forms: as usual, $d_{M}$ denotes the exterior derivative, while $\delta_{M}$ is the codifferential, defined with the conventions of [1] (also used in [25]) in which $\square_{\boldsymbol{M}}=-\left(\delta_{\boldsymbol{M}} d_{\boldsymbol{M}}+d_{\boldsymbol{M}} \delta_{\boldsymbol{M}}\right)$, [i.e., minus the Laplace-de Rham operator], which agrees with the action of $g^{a b} \nabla_{a} \nabla_{b}$ up to lower order terms that vanish on 0 -forms. Thus $\square_{M}$ has metric principal symbol on $p$-forms of any degree and has unique advanced (-) and retarded (+) fundamental solutions $E_{\boldsymbol{M}}^{ \pm}: \Omega_{0}^{p}(\boldsymbol{M}) \rightarrow \Omega^{p}(\boldsymbol{M})$ extending the usual notation for 0-forms. A key property is that the exterior derivative and coderivative commute with $\square_{M}$ and hence $E_{M}^{ \pm}$(or more precisely intertwine their actions on forms of adjacent rank).

For any $\boldsymbol{M} \in$ Loc, let $\mathscr{L}_{0}(\boldsymbol{M})$ be the space of $\phi \in C^{\infty}(\boldsymbol{M})$ such that $\square_{\boldsymbol{M}} \phi=0$ and obeying the following conditions: (A) there is at least one locally constant function $c$ so that the support of $\phi-c$ has compact intersection with all Cauchy surfaces:7 (B) the constraint

$$
\int_{\Sigma} \nabla_{n} \phi d \Sigma=0
$$

\footnotetext{
${ }^{7}$ The function $c$ is unique if and only if $\boldsymbol{M}$ has purely noncompact Cauchy surfaces.
} 
holds on each smooth spacelike Cauchy surface $\Sigma$ of each component of $\boldsymbol{M}$, where $n^{a}$ is the unit future-pointing normal field to $\Sigma$. It is enough to verify conditions (A) and (B) for any particular choices of Cauchy surface to deduce that they hold in general: for (B) this follows using the field equation and divergence theorem, in conjunction with the support properties of (A). Conditions (A) and (B) together entail that the usual formula for the symplectic product $\sigma_{M}$ gives a well-defined convergent integral on solutions in $\mathscr{L}_{0}(\boldsymbol{M})$, although it is now degenerate as the locally constant solutions have vanishing symplectic product with all other solutions - this is exactly the content of Eq. (4.1), which can also be interpreted as the vanishing of the Noether charge associated with the $\phi \mapsto \phi+c$ invariance in each component of $\boldsymbol{M}$. Next, we define the linear equivalence relation $\phi \sim \phi^{\prime}$ on $\mathscr{L}_{0}(\boldsymbol{M})$ to mean that $\phi-\phi^{\prime}$ is locally constant, and then take the quotient $\mathscr{J}(\boldsymbol{M})=\mathscr{L}_{0}(\boldsymbol{M}) / \sim$ as the classical phase space of the theory. Condition (B) guarantees that the symplectic form descends to an antisymmetric bilinear form $\sigma_{0} \boldsymbol{M}$ on the equivalence classes in $\mathscr{J}(\boldsymbol{M})$ and is readily seen to be nondegenerate: if $\sigma_{0 \boldsymbol{M}}\left([\phi],\left[\phi^{\prime}\right]\right)=0$ for all $\left[\phi^{\prime}\right] \in \mathscr{J}(\boldsymbol{M})$, then $\sigma_{\boldsymbol{M}}\left(\phi, \phi^{\prime}\right)=0$ for all $\phi^{\prime} \in \mathscr{L}(\boldsymbol{M})$, where $\phi$ is a representative of $[\phi]$ in $\mathscr{L}(\boldsymbol{M})$; it follows that $\phi$ and hence $[\phi]$ vanish, because $\sigma_{\boldsymbol{M}}$ is weakly nondegenerate. Thus $\mathscr{J}(\boldsymbol{M})$, equipped with $\sigma_{0} \boldsymbol{M}$ and complex conjugation defines a weakly nondegenerate object of preSympl $\mathrm{I}_{\mathbb{C}}$.

The covariance of this theory is easily established using the following result.

Lemma 4.1 Suppose $\psi: \boldsymbol{M} \rightarrow \boldsymbol{N}$ in Loc. Then (a) $\mathscr{L}(\psi)\left(\mathscr{L}_{\text {l.c. }}(\boldsymbol{M})\right) \subset \mathscr{L}_{\text {l.c. }}(\boldsymbol{N})$; (b) if $\phi \in \mathscr{L}(\boldsymbol{M})$ obeys condition $(B)$ in $\boldsymbol{M}$ (whereupon $[\phi] \in \mathscr{J}(\boldsymbol{M})$ ) then $\mathscr{L}(\psi) \phi$ also obeys condition $(B)$ in $\boldsymbol{N}$ and hence $[\mathscr{L}(\psi) \phi] \in \mathscr{J}(\boldsymbol{N})$.

Proof (a) Take $\chi \in C^{\infty}(\boldsymbol{M})$ so that $d \chi=0$ outside a neighbourhood of a Cauchy surface in $\boldsymbol{M}$ and so that $\eta=E_{\boldsymbol{M}} \square_{\boldsymbol{M}} \chi \eta$ for any $\eta \in \mathscr{L}(\boldsymbol{M})$. Then $\mathscr{L}(\psi) \eta=E_{\boldsymbol{N}} \psi_{*} \square_{\boldsymbol{M}} \chi \eta$ and using well-known intertwining relations,

$$
d \mathscr{L}(\psi) \eta=E_{\boldsymbol{N}}^{(1)} d \psi_{*} \square_{\boldsymbol{M}} \chi \eta=E_{\boldsymbol{N}}^{(1)} \psi_{*} \square_{\boldsymbol{M}}^{(1)} d \chi \eta,
$$

where $d$ is the exterior derivative and $E^{(1)}, \square^{(1)}$ are the 1 -form analogues of $E$, $\square$. If $\eta \in$ $\mathscr{L}_{\text {l.c. }}(\boldsymbol{M})$ then $d \chi \eta$ has compact support 8 and we get $\psi_{*} \square_{M}^{(1)} d \chi \eta=\square_{\boldsymbol{N}}^{(1)} \psi_{*} d \chi \eta$, whereupon it is clear that $d \mathscr{L}(\psi) \eta=0$ so $\mathscr{L}(\psi) \eta \in \mathscr{L}_{\text {l.c. }}(\boldsymbol{N})$.

(b) We observe that $\phi=E_{\boldsymbol{M}} f\left(f \in C_{0}^{\infty}(\boldsymbol{M})\right)$ obeys condition (B) if and only if for each component $\boldsymbol{B}$ of $\boldsymbol{M}$, with Cauchy surface $\Sigma_{\boldsymbol{B}}$,

$$
0=\int_{\Sigma_{B}} \nabla_{n} \phi d \Sigma=\int_{B} f d \operatorname{vol}_{B}
$$

using [19, Lem. A.1] applied to the smooth constant solution 1. Thus if $\phi=E_{M} f$ obeys condition (B) then $\mathscr{L}(\psi) \phi=E_{\boldsymbol{N}} \psi_{*} f$ with $\int_{\boldsymbol{C}} \psi_{*} f d \mathrm{vol}=0$ in each component $\boldsymbol{C}$ of $\boldsymbol{N}$, so $\mathscr{L}(\psi) \phi$ obeys condition (B) in $\boldsymbol{N}$.

Given this result, for $\psi: \boldsymbol{M} \rightarrow \boldsymbol{N}$ it is possible to define $\mathscr{J}(\psi): \mathscr{J}(\boldsymbol{M}) \rightarrow \mathscr{J}(\boldsymbol{N})$ by $\mathscr{J}(\psi)[\phi]=[\mathscr{L}(\psi) \phi]$, where $\phi$ is a representative lying in $\mathscr{L}(\boldsymbol{M})$. (Note that $\mathscr{L}(\psi) \phi$ is

\footnotetext{
${ }^{8}$ Recall that $\eta$ can only be nonzero on components of $\boldsymbol{M}$ with compact Cauchy surfaces.
} 
then a representative of $\mathscr{J}(\psi)[\phi]$ in $\mathscr{L}(\boldsymbol{N})$.) This is well-defined because if $[\phi]=\left[\phi^{\prime}\right]$ with both $\phi, \phi^{\prime} \in \mathscr{L}(\boldsymbol{M})$, then $\phi-\phi^{\prime} \in \mathscr{L}_{\text {l.c. }}(\boldsymbol{M})$ and hence $\mathscr{L}(\psi) \phi \sim \mathscr{L}(\psi) \phi^{\prime}$ by Lemma 4.1(a). Moreover, as

$$
\sigma_{0 N}\left(\mathscr{J}(\psi)[\phi], \mathscr{J}(\psi)\left[\phi^{\prime}\right]\right)=\sigma_{N}\left(\mathscr{L}(\psi) \phi, \mathscr{L}(\psi) \phi^{\prime}\right)=\sigma_{M}\left(\phi, \phi^{\prime}\right)=\sigma_{0 M}\left([\phi],\left[\phi^{\prime}\right]\right),
$$

it is clear that $\mathscr{J}(\psi): \mathscr{J}(\boldsymbol{M}) \rightarrow \mathscr{J}(\boldsymbol{N})$ in Sympl, so $\mathscr{J}(\psi)$ is necessarily injective. The functorial property of $\mathscr{L}$ induces the corresponding property for $\mathscr{J}$. Hence $\mathscr{J}$ is indeed a functor from Loc to Sympl and is easily shown to have the timeslice property: if $\psi$ is Cauchy, it is clear that $[\phi] \mapsto\left[\mathscr{L}(\psi)^{-1} \phi\right]$ is inverse to $\mathscr{J}(\psi)$ - one need only check that $\mathscr{L}(\psi)$ maps $\mathscr{L}_{\text {l.c. }}(\boldsymbol{M})$ surjectively onto $\mathscr{L}_{\text {l.c. }}(\boldsymbol{N})$ for Cauchy $\psi$.

From the definition of $\mathscr{J}(\psi)$ it follows immediately that the kinematic subspaces are given as

$$
\mathscr{J}^{\operatorname{kin}}(\boldsymbol{M} ; O)=\left[\mathscr{L}^{\mathrm{kin}}(\boldsymbol{M} ; O)\right]
$$

for nonempty $O \in \mathscr{O}(\boldsymbol{M})$. Furthermore, the relative Cauchy evolution is easily seen to be

$$
\operatorname{rce}_{0}[\boldsymbol{h}][\phi]=\left[\operatorname{rce}_{\boldsymbol{M}}[\boldsymbol{h}] \phi\right]
$$

for $\boldsymbol{h} \in H(\boldsymbol{M})$, where $\phi$ is a representative in $\mathscr{L}(\boldsymbol{M})$ of $[\phi]$, and we use rce $_{0}$ and rce in place of $\operatorname{rce}^{(\mathscr{J})}$ and rce $^{(\mathscr{L})}$ to unburden the notation.

Proposition 4.2 For any compact $K \subset \boldsymbol{M}$, and any $O \in \mathscr{O}(\boldsymbol{M})$ we have

$$
\mathscr{J}^{\bullet}(\boldsymbol{M} ; K)=\left[\mathscr{L}^{\bullet}(\boldsymbol{M} ; K)\right], \quad \text { and } \quad \mathscr{J}^{\mathrm{dyn}}(\boldsymbol{M} ; O)=\left[\mathscr{L}^{\mathrm{dyn}}(\boldsymbol{M} ; O)\right] .
$$

If, additionally, $O$ is nonempty, we also have $\mathscr{J}^{\operatorname{kin}}(\boldsymbol{M} ; O) \subset \mathscr{J}^{\operatorname{dyn}}(\boldsymbol{M} ; O)$.

Proof: We note that $[\phi] \in \mathscr{J}^{\bullet}(\boldsymbol{M} ; K$ ) (with $\phi$ a representative in $\mathscr{L}(\boldsymbol{M})$ ) if and only if $\operatorname{rce}_{\boldsymbol{M}}[\boldsymbol{h}] \phi \sim \phi$ for all $\boldsymbol{h} \in H\left(\boldsymbol{M} ; K^{\perp}\right)$. But as both $\phi$ and rce $_{\boldsymbol{M}}[\boldsymbol{h}] \phi$ have compact support on Cauchy surfaces, their difference must therefore be an element of $\mathscr{L}_{\text {l.c. }}(\boldsymbol{M})$. Accordingly the finite dimensional subspace $\mathbb{C} \phi+\mathscr{L}_{\text {l.c. }}(\boldsymbol{M})$ is invariant under all such rce $_{\boldsymbol{M}}[\boldsymbol{h}]$, and by Prop. 3.7 is therefore contained in $\mathscr{L}^{\bullet}(\boldsymbol{M} ; K)$. In particular, $\phi \in \mathscr{L}^{\bullet}(\boldsymbol{M} ; K)$. As the reverse inclusion is trivial, the first equality is proved; the second follows immediately on taking the linear spans. Finally, we use the above results to compute

$$
\mathscr{J}^{\mathrm{kin}}(\boldsymbol{M} ; O)=\left[\mathscr{L}^{\mathrm{kin}}(\boldsymbol{M} ; O)\right] \subset\left[\mathscr{L}^{\mathrm{dyn}}(\boldsymbol{M} ; O)\right]=\mathscr{J}^{\mathrm{dyn}}(\boldsymbol{M} ; O)
$$

where the inclusion follows from Lem. 3.3 .

The spacetime dimension now enters in an essential way. In dimensions $n>2$, $\mathscr{L}^{\text {dyn }}(\boldsymbol{M} ; O)$ differs from $\mathscr{L}^{\text {kin }}(\boldsymbol{M} ; O$ ) (for nonempty $O \in \mathscr{O}(\boldsymbol{M})$ ) only by locally constant solutions, which are annihilated by the quotient, thus giving dynamical locality:

$$
\mathscr{J}^{\mathrm{dyn}}(\boldsymbol{M} ; O)=\left[\mathscr{L}^{\mathrm{dyn}}(\boldsymbol{M} ; O)\right]=\left[\mathscr{L}^{\mathrm{kin}}(\boldsymbol{M} ; O)+\mathscr{L}_{\text {l.c. }}(\boldsymbol{M})\right]=\left[\mathscr{L}^{\mathrm{kin}}(\boldsymbol{M} ; O)\right]=\mathscr{J}^{\mathrm{kin}}(\boldsymbol{M} ; O) .
$$


In $n=2$ dimensions, however, there is an added complication. First, consider the case in which $O \in \mathscr{O}_{0}(\boldsymbol{M})$ for $\boldsymbol{M} \in \mathrm{Loc}_{0}$. If $K \subset O$ is the closure of the base of a multidiamond, then there is a smooth spacelike Cauchy surface $\Sigma$ for $O$ that contains $K$. As a connected one-dimensional paracompact manifold, $\Sigma$ is homeomorphic to either $\mathbb{R}$ or $S^{1}$; in either case, as $K$ must have nonempty causal complement, it is evident that we may find a connected, contractible compact set $\tilde{K}$ so that $K \subset \tilde{K} \subset \Sigma$. Then it is clear that

$$
\mathscr{J}^{\bullet}(\boldsymbol{M} ; K) \subset \mathscr{J}^{\bullet}(\boldsymbol{M} ; \tilde{K}) \subset \mathscr{J}^{\operatorname{kin}}(\boldsymbol{M} ; O)
$$

and as $\mathscr{J}^{\text {dyn }}(\boldsymbol{M} ; O)$ is generated over such $K$, we have the inclusion $\mathscr{J}^{\text {dyn }}(\boldsymbol{M} ; K) \subset$ $\mathscr{J}^{\operatorname{kin}}(\boldsymbol{M} ; O)$. Together with the last statement of Prop. 4.2, this establishes dynamical locality for $\mathscr{J}$ in $\mathrm{LCT}_{0}$, i.e., when regarded as a theory on the category of connected spacetimes, Loc $_{0}$.

On the other hand, we can also see that dynamical locality fails when disconnected spacetimes are permitted, i.e., in LCT. Let $\boldsymbol{M}_{0}$ be two-dimensional Minkowski space, with standard $(t, x)$ coordinates and metric $d t^{2}-d x^{2}$, and let $O$ be the Cauchy development of the set $B=\{(0, x): a<|x|<2 a\}$ for some $a>0$. The causal complement $B^{\perp}$ consists of the Cauchy development of $(-\infty,-2 a) \cup(-a, a) \cup(2 a, \infty)$, which has three connected components. Now let $f \in C_{0}^{\infty}(\mathbb{R})$ be such that $f \equiv 1$ on $(-a, a)$ and $f \equiv 0$ outside $(-2 a, 2 a)$. Then $\phi(t, x)=\frac{1}{2}(f(x-t)+f(x+t))$ is a solution taking the value 1 inside the Cauchy development of $(-a, a)$ and vanishing in the other two components of $B^{\perp}$. This solution is invariant under relative Cauchy evolutions induced by metric perturbations supported in $B^{\perp}$ but is not locally constant; accordingly, $[\phi]$ is a nonzero element of $\mathscr{J} \bullet\left(\boldsymbol{M}_{0} ; B\right) \subset \mathscr{J}^{\text {dyn }}\left(\boldsymbol{M}_{0} ; O\right)$. However, $[\phi]$ is not an element of $\mathscr{J}^{\operatorname{kin}}\left(\boldsymbol{M}_{0} ; O\right)$, because the pull-back $\iota_{M ; O}^{*} \phi$ of $\phi$ to $O$ cannot be reduced to a solution of compact support on Cauchy surfaces (of $O$ ) by adding a locally constant function. Thus $\mathscr{J}^{\operatorname{kin}}\left(\boldsymbol{M}_{0} ; O\right)$ is a proper subspace of $\mathscr{J}^{\text {dyn }}\left(\boldsymbol{M}_{0} ; O\right)$, and dynamical locality fails.

Summarising:

Theorem 4.3 The theory $\mathscr{J}$ is dynamically local in $\mathrm{LCT}_{0}$ for all dimensions $n \geq 2$. It is dynamically local in LCT for all dimensions $n \geq 3$, but not in dimension $n=2$.

Moreover, the above discussion shows that $\mathscr{J}$ obeys conditions $(\mathscr{L} 1-\mathscr{L} 4)$ in all dimensions $n \geq 2$, and whether formulated in LCT or $\mathrm{LCT}_{0}$. For $(\mathscr{L} 1)$ and $(\mathscr{L} 2)$ hold because the relative Cauchy evolution of $\mathscr{J}$ is inherited from that of $\mathscr{L}$, and the classical stressenergy tensor of the massless scalar field is independent of the choice of representative in equivalence classes modulo locally constant functions; likewise, $(\mathscr{L} 3)$ follows from the extended locality of $\mathscr{L}$, while $(\mathscr{L} 4)$ holds by the first equation in Prop. 4.2 .

The failure of dynamical locality for $\mathscr{J}$ in LCT for dimension $n=2$ suggests the need for further work. The cause of the defect is evidently connected to the presence of topological charge; we conjecture that it can be addressed by admitting topological charge as an additional background feature of spacetimes. At any rate, it seems clear that when dynamical locality fails, it does so for interesting reasons.

Finally, we give a technical result that will be used later on. 
Lemma 4.4 The map $\Omega_{0}^{1}(\boldsymbol{M}) \ni \omega \mapsto\left[E_{\boldsymbol{M}} \delta_{\boldsymbol{M}} \omega\right]$ is a linear surjection onto $\mathscr{J}(\boldsymbol{M})$ with kernel ker $\delta_{\boldsymbol{M}} \cap \Omega_{0}^{1}(\boldsymbol{M})+\left(\Omega_{0}^{1}(\boldsymbol{M}) \cap d_{\boldsymbol{M}} C^{\infty}(\boldsymbol{M})\right)$.

Proof: First, suppose $\omega \in \Omega_{0}^{1}(\boldsymbol{M})$ and set $\phi=E_{\boldsymbol{M}} \delta_{\boldsymbol{M}} \omega$. Let $\boldsymbol{C}$ be any connected component of $\boldsymbol{M}$, let $\Sigma_{\boldsymbol{C}}$ be a Cauchy surface for $\boldsymbol{C}$ with unit future-pointing normal $\boldsymbol{n}$, and $1_{\boldsymbol{C}}$ the locally constant function on $\boldsymbol{M}$ that takes the value 1 on $\boldsymbol{C}$ and 0 otherwise. Then

$$
\int_{\Sigma_{C}} \nabla_{n} \phi d \Sigma_{C}=\sigma_{M}\left(E_{M} \delta_{M} \omega, 1_{C}\right)=\int_{M} 1_{C} \delta_{M} \omega d \operatorname{vol}_{M}=\left.\int_{C} \delta_{C} \omega\right|_{C} d \operatorname{vol}_{C}=0,
$$

by [19, Lem. A.1] (because $1_{\boldsymbol{C}}$ is a smooth solution while $\delta_{\boldsymbol{M}} \omega \in C_{0}^{\infty}(\boldsymbol{M})$ ) and as $\boldsymbol{C}$ was arbitrary, we have $\left[E_{\boldsymbol{M}} \delta_{\boldsymbol{M}} \omega\right] \in \mathscr{J}(\boldsymbol{M})$; the map is evidently linear. To check surjectivity, given any element of $\mathscr{J}(\boldsymbol{M})$ choose a representative $\phi$ with compact support on Cauchy surfaces and write $\phi=E_{\boldsymbol{M}} f$ for some $f \in C_{0}^{\infty}(\boldsymbol{M})$. Then, with $\boldsymbol{C}, \Sigma_{\boldsymbol{C}}, 1_{\boldsymbol{C}}$ as before,

$$
\left.\int_{C} f\right|_{C} d \operatorname{vol}_{C}=\sigma_{C}\left(\left.E_{C} f\right|_{C}, 1\right)=\int_{\Sigma_{C}} \nabla_{n} \phi d \Sigma_{C}=0,
$$

from which we may deduce that $\left.f\right|_{\boldsymbol{C}} \in \delta_{\boldsymbol{C}} \Omega_{0}^{1}(\boldsymbol{C})$ for each $\boldsymbol{C}$ and hence $f \in \delta_{\boldsymbol{M}} \Omega_{0}^{1}(\boldsymbol{M})$ by a standard result on the compact cohomology group of highest degree for connected manifolds, (see, e.g., Theorem 7.5.19(i) in [1]). Accordingly, surjectivity holds.

Next, suppose $\omega \in \Omega_{0}^{1}(\boldsymbol{M})$ and $\left[E_{\boldsymbol{M}} \delta_{\boldsymbol{M}} \omega\right]=0$. Then $0=d_{\boldsymbol{M}} E_{\boldsymbol{M}} \delta_{\boldsymbol{M}} \omega=E_{\boldsymbol{M}} d_{\boldsymbol{M}} \delta_{\boldsymbol{M}} \omega$ and hence

$$
d_{M} \delta_{M} \omega=\square_{M} \beta
$$

for some $\beta \in \Omega_{0}^{1}(\boldsymbol{M})$. In particular, $\beta$ vanishes to the past, so we may solve for $\beta$ by applying the retarded fundamental solution for 1 -forms,

$$
\beta=E_{M}^{+} d_{M} \delta_{M} \omega=d_{M} E_{M}^{+} \delta_{M} \omega \in \Omega_{0}^{1}(\boldsymbol{M}) \cap d_{\boldsymbol{M}} C^{\infty}(\boldsymbol{M})
$$

(equally, we could have used the advanced fundamental solution). On the other hand, Eq. (4.2) also implies

$$
\square_{\boldsymbol{M}} \delta_{\boldsymbol{M}} \omega=-\delta_{\boldsymbol{M}} d_{\boldsymbol{M}} \delta_{\boldsymbol{M}} \omega=-\delta_{\boldsymbol{M}} \square_{\boldsymbol{M}} \beta=-\square_{\boldsymbol{M}} \delta_{\boldsymbol{M}} \beta
$$

and hence $\delta_{M} \omega=-\delta_{M} \beta$ (as both have compact support). Thus $\omega=-\beta+\kappa$ for some $\kappa \in \operatorname{ker} \delta_{M}$ and $\beta \in \Omega_{0}^{1}(\boldsymbol{M}) \cap d_{\boldsymbol{M}} C^{\infty}(\boldsymbol{M})$. Finally, suppose that $\omega$ takes this form, with $\beta=-d_{\boldsymbol{M}} \chi \in \Omega_{0}^{1}(\boldsymbol{M}), \chi \in C^{\infty}(\boldsymbol{M})$. Then

$$
d_{M} E_{M} \delta_{M} \omega=E_{M} d_{M} \delta_{M} d_{M} \chi=-E_{M} \square_{M} d_{M} \chi=0
$$

(recall that $d_{\boldsymbol{M}} \chi$ has compact support) so $\left[E_{\boldsymbol{M}} \delta_{\boldsymbol{M}} \omega\right]=[0]$. 


\section{Quantized theory: smeared fields}

In this section, we describe how weakly nondegenerate dynamically local classical theories, valued in preSympl $\mathbb{C}$ and obeying the additional conditions $(\mathscr{L} 1-\mathscr{L} 4)$, can be quantized as the 'infinitesimal Weyl algebra' (cf. [3]) to obtain a dynamically local quantum field theory. For the Klein-Gordon case, this is the usual *-algebra of smeared fields. We begin by describing this quantization method in a form that will be convenient for our purposes. We will also make contact with other standard presentations of the theory.

\subsection{Quantization functor}

The infinitesimal Weyl algebra quantization of a presymplectic space $(V, \sigma, C) \in$ preSympl $_{\mathbb{C}}$ is given by the unital $*$-algebra $\mathscr{Q}(V, \sigma, C)$, whose underlying complex vector space is the symmetric tensor vector space over $V$,

$$
\mathscr{Q}(V, \sigma, C)=\Gamma_{\odot}(V) \stackrel{\text { def }}{=} \bigoplus_{n \in \mathbb{N}_{0}} V^{\odot n},
$$

equipped with a product such that

$$
u^{\odot m} \cdot v^{\odot n}=\sum_{r=0}^{\min \{m, n\}}\left(\frac{i \sigma(u, v)}{2}\right)^{r} \frac{m ! n !}{r !(m-r) !(n-r) !} S\left(u^{\otimes(m-r)} \otimes v^{\otimes(n-r)}\right),
$$

where $S$ denotes symmetrisation, and a $*$-operation defined by $\left(u^{\odot n}\right)^{*}=(C u)^{\odot n}$; both operations being extended by (anti-)linearity to general elements of $\Gamma_{\odot}(V)$. In the above, all tensor products and direct sums are algebraic - we do not complete in any topology - and by convention $u^{\odot}=1 \in V^{\odot 0}=\mathbb{C}, f^{\otimes 0}=\mathrm{id}_{\mathbb{C}}$. The product may be summarised via the Weyl relations $W(\lambda u) W(\mu v)=e^{-i \lambda \mu \sigma(u, v) / 2} W(\lambda u+\mu v)$, understood as relations between the formal power series

$$
W(\lambda u)=\bigoplus_{n=0}^{\infty} \frac{(i \lambda)^{n}}{n !} u^{\odot n}, \quad(u \in V, \lambda \in \mathbb{R}) .
$$

(Here, it is not necessary to demand that $u$ is 'real', in the sense that $u=C u$.)

In addition, given any morphism $f:(V, \sigma, C) \rightarrow\left(V^{\prime}, \sigma^{\prime}, C^{\prime}\right)$ in preSympl $_{\mathbb{C}}$, we define

$$
\mathscr{Q}(f)=\Gamma_{\odot}(f)=\bigoplus_{n \in \mathbb{N}_{0}}^{\infty} f^{\odot n}
$$

Proposition 5.1 Equations (5.1), (5.2) and (5.3) define a functor $\mathscr{Q}:$ preSympl $_{\mathbb{C}} \rightarrow$ Alg. If $(V, \sigma, C)$ is weakly nondegenerate, then $\mathscr{Q}(V, \sigma, C)$ is simple.

The proof of this result is largely a matter of assembling standard results - it will be given below for completeness. Before that, we make a number of remarks. 
For obvious reasons, we refer to $\mathscr{Q}$ as a quantization functor. Given any classical theory $\mathscr{L}:$ Loc $\rightarrow$ preSympl $_{\mathbb{C}}$, we obtain a quantum theory $\mathscr{A}=\mathscr{Q} \circ \mathscr{L} ;$ if $\mathscr{L}$ obeys the timeslice property, then so does $\mathscr{A}$, because functors preserve isomorphisms, and its relative Cauchy evolution is given by

$$
\operatorname{rce}_{M}[\boldsymbol{h}]=\mathscr{Q}\left(\operatorname{rce}_{M}^{(\mathscr{L})}[\boldsymbol{h}]\right)=\bigoplus_{n \in \mathbb{N}_{0}} R_{M}[\boldsymbol{h}]^{\otimes n}
$$

where, to unburden the notation, we have written rce for rce $\mathrm{e}^{(\mathscr{A})}$ and $R$ for rce $\mathrm{e}^{(\mathscr{L})}$. Furthermore, $\mathscr{Q}$ interacts well with the unions in preSympl $\mathbb{C}_{\mathbb{C}}$ and Alg: given a (possibly infinite) family of $C$-invariant subspaces of $V, W_{i}$, then

$$
\bigvee_{i} \Gamma_{\odot}\left(W_{i}\right)=\Gamma_{\odot}\left(\bigvee_{i} W_{i}\right),
$$

where the union on the left-hand side is an algebraic span (in $\mathscr{Q}(V, \sigma, C)$ ), while that on the right is a vector space span in $V$. Inclusion of the left-hand side in the right-hand side is obvious; the reverse inclusion arises from the freedom to form products as well as linear combinations in Alg.

We now turn to the proof of Prop. 5.1, beginning by giving a construction of $\mathscr{Q}(V, \sigma, C)$ that allows its various properties to be established. Let iVect be the category whose objects are pairs $(V, C)$, where $C$ is an antilinear involution on complex vector space $V$, and with morphisms $f:(V, C) \rightarrow\left(V^{\prime}, C^{\prime}\right)$ which are injective linear maps such that $C^{\prime} \circ f=f \circ C$. Then there is a functor $\mathscr{T}:$ iVect $\rightarrow$ Alg which constructs the tensor algebra over given vector spaces:

$$
\mathscr{T}(V)=\bigoplus_{n \in \mathbb{N}_{0}} V^{\otimes n}, \quad \mathscr{T}(f)=\bigoplus_{n \in \mathbb{N}_{0}} f^{\otimes n}
$$

where the product in $\mathscr{T}(V)$ is given by the tensor product and the $*$-operation by

$$
\left(\phi_{1} \otimes \cdots \otimes \phi_{n}\right)^{*}=C\left(\phi_{n}\right) \otimes \cdots \otimes C\left(\phi_{1}\right)
$$

(injectivity of $\mathscr{T}(f)$ follows from injectivity of $f$ after some multilinear algebra - see Appendix $\AA$ for details). As above, all tensor products and direct sums are algebraic.

There is an obvious forgetful functor from preSympl $\mathbb{C}_{\mathbb{C}}$ to iVect, and so $\mathscr{T}$ (we use the same notation for $\mathscr{T}$ and its composition with the forgetful functor) can be defined from preSympl $_{\mathbb{C}} \rightarrow$ Alg. Given any $(V, \sigma, C)$, let $\mathscr{Z}(V, \sigma, C)$ be the two-sided $*$-ideal in $\mathscr{T}(V, \sigma, C)$ generated by elements of form

$$
(-i \sigma(u, v), 0, u \otimes v-v \otimes u, 0, \ldots) \quad(u, v \in V)
$$

and write $\mathscr{Q}(V, \sigma, C)$ for the quotient $\mathscr{T}(V, \sigma, C) / \mathscr{Z}(V, \sigma, C)$. If $f:(V, \sigma, C) \rightarrow\left(V^{\prime}, \sigma^{\prime}, C^{\prime}\right)$ then $\mathscr{T}(f)$ maps $\mathscr{Z}(V, \sigma, C)$ into $\mathscr{Z}\left(V^{\prime}, \sigma^{\prime}, C^{\prime}\right)$, which induces a unital $*$-homomorphism $\mathscr{Q}(f): \mathscr{Q}(V, \sigma, C) \rightarrow \mathscr{Q}\left(V^{\prime}, \sigma^{\prime}, C^{\prime}\right)$.

As it is clear that $\mathscr{Z}(V, \sigma, C)$ has trivial intersection with the symmetric subspace of $\mathscr{T}(V, \sigma, C)$, it follows that (i) the quotient algebras are nontrivial; (ii) every element 
$A \in \mathscr{T}(V, \sigma, C)$ has a unique symmetric representative $A_{\odot}$, in the symmetric subspace and that $A=0$ iff $A_{\odot}=0$; (iii) we may therefore identify $\mathscr{Q}(V, \sigma, C)$ as a vector space with $\Gamma_{\odot}(V)$ (as in (5.1)) and any morphism $\mathscr{Q}(f)\left(f:(V, \sigma, C) \rightarrow\left(V^{\prime}, \sigma^{\prime}, C^{\prime}\right)\right)$ may be identified as a linear map with the restriction of $\mathscr{T}(f)$ to this subspace (as in (5.3)); (iv) the homomorphisms $\mathscr{Q}(f)$ are therefore injective and hence define Alg-morphisms. It is clear that $\mathscr{Q}$ inherits functoriality from $\mathscr{T}$.

To complete the proof of Prop. 5.1, we need to verify the product formula (5.2) and show that $\mathscr{Q}(V, \sigma, C)$ is simple when $(V, \sigma, C)$ is weakly nondegenerate. The latter follows from Scholium 7.1 in [3], while the former requires a tedious calculation with commutators if written explicitly. However, the mere existence of such an argument indicates that the question is purely one of combinatorics, and can be resolved by examining the case in which $V$ is of dimension 2, $\sigma$ is nondegenerate, $u=\lambda e_{1}, v=\mu e_{2}$, for $\lambda, \mu \in \mathbb{R}$, where $\sigma\left(e_{1}, e_{2}\right)=1$ and $C e_{i}=e_{i}$. Invoking a Fock representation (e.g., with respect to the Hilbert space norm in which the $e_{i}$ are orthonormal) the Weyl operators may be obtained as convergent power series on a domain of analytic vectors and the required product may be read off as a consequence of the Weyl relations.

To conclude this discussion, we note that our construction is equivalent to a more familiar quantization of the scalar field (and similar Bose free fields). Let $\mathscr{L}_{\mathbb{C}}$ be the complex Klein-Gordon theory (with mass $m \geq 0$ ), with corresponding quantum field theory $\mathscr{A}=\mathscr{Q} \circ \mathscr{L}_{\mathbb{C}}$. Now, for each $f \in C_{0}^{\infty}(\boldsymbol{M})$ let

$$
\Phi_{\boldsymbol{M}}(f)=\left(0, E_{\boldsymbol{M}} f, 0, \ldots\right) \in \mathscr{A}(\boldsymbol{M})=\Gamma_{\odot}\left(\mathscr{L}_{\mathbb{C}}(\boldsymbol{M})\right) .
$$

As $\mathscr{L}_{\mathbb{C}}(\boldsymbol{M})$ is precisely the range of $E_{\boldsymbol{M}}$ on $C_{0}^{\infty}(\boldsymbol{M})$, it is easy to see that the $\Phi_{\boldsymbol{M}}(f)$ generate $\mathscr{A}(\boldsymbol{M})$ and obey the relations:

- $f \mapsto \Phi_{M}(f)$ is complex linear

- $\Phi_{M}(f)^{*}=\Phi_{M}(\bar{f})$

- $\Phi_{M}\left(P_{M} f\right)=0$

- $\left[\Phi_{M}(f), \Phi_{M}\left(f^{\prime}\right)\right]=i E_{M}\left(f, f^{\prime}\right) \mathbf{1}$

for all $f, f^{\prime} \in C_{0}^{\infty}(\boldsymbol{M})$. In fact, owing to simplicity of $\mathscr{A}(\boldsymbol{M})$, it may be completely characterized by these generators and relations. Moreover, for any morphism $\psi: \boldsymbol{M} \rightarrow \boldsymbol{N}$, we have

$$
\mathscr{A}(\psi) \Phi_{M}(f)=\left(0, \mathscr{L}_{\mathbb{C}}(\psi) E_{M} f, 0, \ldots\right)=\left(0, E_{\boldsymbol{N}} \psi_{*} f, 0, \ldots\right)=\Phi_{\boldsymbol{N}}\left(\psi_{*} f\right) .
$$

In this sense, $\Phi$ may be regarded as a natural transformation between the functor $\mathscr{D}:$ Loc $\rightarrow$ Vect, with $\mathscr{D}(\boldsymbol{M})=C_{0}^{\infty}(\boldsymbol{M}), \mathscr{D}(\psi)=\psi_{*}$, and the functor $\mathscr{A}$, after the latter is composed with a forgetful functor to the category of vector spaces. This is the understanding of 'quantum fields as natural transformations' first articulated by BFV.

The quantized massless current, $\mathscr{C}=\mathscr{Q} \circ \mathscr{J}$, may be treated in the same way. To each $\omega \in \Omega_{0}^{1}(\boldsymbol{M})$, we assign $\mathrm{J}_{\boldsymbol{M}}(\omega)=\left(0,\left[E_{\boldsymbol{M}} \delta_{\boldsymbol{M}} \omega\right], 0, \ldots\right) \in \Gamma_{\odot}(\mathscr{J}(\boldsymbol{M}))=\mathscr{C}(\boldsymbol{M})$. The surjectivity result in Lemma 4.4 establishes that these elements generate $\mathscr{C}(\boldsymbol{M})$; moreover, they clearly satisfy the relations 
- $\Omega_{0}^{1}(\boldsymbol{M}) \ni \omega \mapsto \mathrm{J}_{\boldsymbol{M}}(\omega)$ is complex linear

- $\mathrm{J}_{M}(\omega)^{*}=\mathrm{J}_{M}(\bar{\omega})$, for all $\omega \in \Omega_{0}^{1}(\boldsymbol{M})$

- $\mathrm{J}_{\boldsymbol{M}}(\omega)=0$ for all $\omega \in \Omega_{0}^{1}(\boldsymbol{M}) \cap \operatorname{ker} \delta_{\boldsymbol{M}}+\Omega_{0}^{1}(\boldsymbol{M}) \cap d_{\boldsymbol{M}} C^{\infty}(\boldsymbol{M})$

- $\left[J_{M}(\omega), J_{M}\left(\omega^{\prime}\right)\right]=i E_{\boldsymbol{M}}\left(\delta_{\boldsymbol{M}} \omega, \delta_{\boldsymbol{M}} \omega^{\prime}\right) \mathbf{1}$ for all $\omega, \omega^{\prime} \in \Omega_{0}^{1}(\boldsymbol{M})$.

The last of these holds because

$$
\sigma_{0 \boldsymbol{M}}\left(\left[E_{\boldsymbol{M}} \delta_{\boldsymbol{M}} \omega\right],\left[E_{\boldsymbol{M}} \delta_{\boldsymbol{M}} \omega^{\prime}\right]\right)=\sigma_{\boldsymbol{M}}\left(E_{\boldsymbol{M}} \delta_{\boldsymbol{M}} \omega, E_{\boldsymbol{M}} \delta_{\boldsymbol{M}} \omega^{\prime}\right)=E_{\boldsymbol{M}}\left(\delta_{\boldsymbol{M}} \omega, \delta_{\boldsymbol{M}} \omega^{\prime}\right) .
$$

Moreover, under any $\psi: \boldsymbol{M} \rightarrow \boldsymbol{N}$ in Loc, we have the naturality property $\mathscr{C}(\psi) \mathrm{J}_{\boldsymbol{M}}(\omega)=$ $\mathrm{J}_{\boldsymbol{N}}\left(\psi_{*} \omega\right)$ by a similar calculation to that used in the standard scalar field case. Although it is not immediately obvious, the third relation above is compatible with this map, because $\mathscr{C}$ is known to be a functor. A direct proof can be given, but will not be done here.

\subsection{Dynamical locality}

Suppose that $\mathscr{L}:$ Loc $\rightarrow$ preSympl $_{\mathbb{C}}$ is a weakly nondegenerate locally covariant theory, obeying the general conditions $(\mathscr{L} 1-\mathscr{L} 4)$ stated in Sect. 3 , but which is not necessarily dynamically local. 9 As shown above, all these assumptions hold for the massive minimally coupled field and the massless current and are expected to hold for the dynamically local linear Bose fields of interest. At any rate, as we will see, these statements isolate the properties that are necessary to establish dynamical locality of the quantized theory $\mathscr{A}=$ $\mathscr{Q} \circ \mathscr{L}$ in the case that $\mathscr{L}$ is dynamically local.

To begin the discussion, we note that the kinematic net is easily obtained in terms of that of $\mathscr{L}$ : for any nonempty $O \in \mathscr{O}(\boldsymbol{M})$ we have $\mathscr{A}\left(\iota_{\boldsymbol{M} ; O}\right)=\mathscr{Q}\left(\mathscr{L}\left(\iota_{\boldsymbol{M} ; O}\right)\right)$ and hence $\mathscr{A}^{\text {kin }}(\boldsymbol{M} ; O)$ may be identified as the linear subspace

$$
\mathscr{A}^{\mathrm{kin}}(\boldsymbol{M} ; O)=\Gamma_{\odot}\left(\mathscr{L}^{\mathrm{kin}}(\boldsymbol{M} ; O)\right) \subset \mathscr{A}(\boldsymbol{M}) .
$$

One might think that there would be a similar 'abstract nonsense' computation of the dynamical nets, given the close relation between the relative Cauchy evolutions of $\mathscr{L}$ and $\mathscr{A}$. It is certainly true that if $\mathscr{Q}$ were to preserve equalizers, intersections and unions, then the dynamical net could be computed in this way. However $\mathscr{Q}$ does not preserve equalizer:10 and we must calculate the dynamical subalgebras directly.

To this end, note that each element $A \in \mathscr{A}(\boldsymbol{M})$ may be associated with a finitedimensional subspace $Y_{A}$ of $\mathscr{L}(\boldsymbol{M})$ in the following way. For each $n \geq 1$, the component

\footnotetext{
${ }^{9}$ In passing, however, we remark that for theories obeying dynamical locality, $(\mathscr{L} 3)$ is equivalent to the absence of nonzero elements invariant under arbitrary relative Cauchy evolution — see Thm 6.5 of [27]. Moreover, in $(\mathscr{L} 2)$, conservation of the stress-energy tensor follows from the arguments given in BFV in the quantum case.

${ }^{10}$ As an example, consider $(V, \sigma, C)$, where $V=\mathbb{C}^{2}$ with a basis $v_{i}(i=1,2)$ obeying $\sigma\left(v_{1}, v_{2}\right)=1$ and $C v_{i}=v_{i}$. Then the map $f\left(a v_{1}+b v_{2}\right)=a v_{2}-b v_{1}(a, b \in \mathbb{C})$ defines an automorphism of $(V, \sigma, C)$ whose equalizer with the identity is trivial. However, $v_{1} \otimes v_{1}+v_{2} \otimes v_{2}$ is a (nonzero) eigenvector of eigenvalue 1 for $\mathscr{Q}(f)$, which therefore has nontrivial equalizer with the identity in $\mathscr{Q}(V, \sigma, C)$.
} 
$A_{n}$ of $A$ in $\mathscr{L}(\boldsymbol{M})^{\odot n}$ may be regarded as a linear map $A_{n}:\left(\mathscr{L}(\boldsymbol{M})^{\otimes(n-1)}\right)^{*} \rightarrow \mathscr{L}(\boldsymbol{M})$ with finite dimensional image, which we call the support subspace of $A_{n}$; the support space $Y_{A}$ of $A$ is defined to be the span of these images for $n \geq 1$, only finitely many of which are nontrivial. (Here, all duals are algebraic.) Moreover, $A \in \Gamma_{\odot}\left(Y_{A}\right)$. Some basic properties of support spaces are summarised in Appendix A. The crucial observation is:

Lemma 5.2 If $A \in \mathscr{A}^{\bullet}(\boldsymbol{M} ; K)$ then the support subspace $Y_{A}$ is invariant under $R_{\boldsymbol{M}}[\boldsymbol{h}]$ for all $\boldsymbol{h} \in H\left(\boldsymbol{M} ; K^{\perp}\right)$ and hence under $F_{\boldsymbol{M}}[\boldsymbol{f}]$ for all $\boldsymbol{f} \in \operatorname{Sym}\left(\boldsymbol{M} ; K^{\perp}\right)$. Hence $\mathscr{A} \bullet(\boldsymbol{M} ; K) \subset$ $\Gamma_{\odot}(\mathscr{L} \bullet(M ; K))$.

Proof: As rce $\boldsymbol{M}[\boldsymbol{h}] A=A$ for all $\boldsymbol{h} \in H\left(\boldsymbol{M} ; K^{\perp}\right)$, we have $\Gamma_{\odot}\left(R_{\boldsymbol{M}}[\boldsymbol{h}]\right) A=A$ for all such $\boldsymbol{h}$, and because the action is diagonal with respect to the direct sum structure, it is clear that the component $A_{n}$ of $A$ in $\mathscr{L}(\boldsymbol{M})^{\odot n}$ obeys $R_{\boldsymbol{M}}[\boldsymbol{h}]^{\otimes n} A_{n}=A_{n}$ for all such $\boldsymbol{h}$. By Lem. A.1, this entails that the support space $Y_{n}$ of $A_{n}$ is invariant under $R_{\boldsymbol{M}}[\boldsymbol{h}]$ for any $n \geq 1$, so the support space $Y_{A}=\bigvee_{n=1}^{\infty} Y_{n}$ is also invariant. The statement regarding invariance under $F_{M}$ follows, given their definition as functional derivatives of the $R_{M}$ with respect to the metric, and we deduce that $Y_{A} \subset \mathscr{L}^{\bullet}(\boldsymbol{M} ; K)$ by Prop. 3.7. Hence $A \in \Gamma_{\odot}\left(Y_{A}\right) \subset \Gamma_{\odot}(\mathscr{L} \bullet(M ; K))$.

Our main result of this section is that the dynamical nets of $\mathscr{A}$ are, after all, related to those of $\mathscr{L}$ by the quantization functor $\mathscr{Q}$. We use the notation $\alpha_{M: X}^{\bullet / d y n / k i n}$ for the inclusions of $\mathscr{A}^{\bullet / \mathrm{dyn} / \mathrm{kin}}(\boldsymbol{M} ; X)$ in $\mathscr{A}(\boldsymbol{M})$, and $\lambda_{\boldsymbol{M} ; X}^{\bullet / \mathrm{dyn} / \mathrm{kin}}$ for the corresponding morphisms in the theory $\mathscr{L}$.

Theorem 5.3 Let $\mathscr{L}:$ Loc $\rightarrow$ preSympl $_{\mathbb{C}}$ be any weakly nondegenerate theory obeying

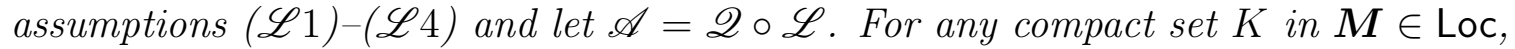

$$
\mathscr{A}^{\bullet}(\boldsymbol{M} ; K)=\Gamma_{\odot}\left(\mathscr{L}^{\bullet}(\boldsymbol{M} ; K)\right),
$$

as vector spaces, i.e., $\alpha_{\boldsymbol{M} ; K}^{\bullet} \cong \mathscr{Q}\left(\lambda_{\boldsymbol{M} ; K}\right)$. For any nonempty $O \in \mathscr{O}(\boldsymbol{M})$,

$$
\mathscr{A}^{\mathrm{dyn}}(\boldsymbol{M} ; O)=\Gamma_{\odot}\left(\mathscr{L}^{\mathrm{dyn}}(\boldsymbol{M} ; O)\right),
$$

i.e., $\alpha_{\boldsymbol{M} ; K}^{\mathrm{dyn}} \cong \mathscr{Q}\left(\lambda_{\boldsymbol{M} ; K}^{\mathrm{dyn}}\right)$. Hence if, additionally, $\mathscr{L}$ is dynamically local, then $\mathscr{A}$ is dynamically local.

Proof. The inclusion of the left-hand side of (5.5) in the right-hand side is established in Lem. 5.2. The reverse inclusion is immediate from (5.4) and the definition of $\mathscr{L} \bullet(\boldsymbol{M} ; K)$. The second part follows from the fact that $\mathscr{Q}$ preserves unions.

Our application is then immediate.

Corollary 5.4 The infinitesimal Weyl-algebra quantizations of (a) the Klein-Gordon theory for $m>0$ in any dimension $n \geq 2$, as a theory on Loc or Loc L $_{0}$ and (b) the theory of the massless current in any dimension $n \geq 2$ as a theory on $\operatorname{Loc}_{0}$, or $n \geq 3$ as a theory on Loc, are dynamically local. The infinitesimal Weyl-algebra quantizations of (c) the massless Klein-Gordon theory in any dimension $n \geq 2$, and as a theory on either Loc $_{0}$ or Loc, and (d) the massless current in dimension $n=2$ as a theory on Loc, are not dynamically local. 
For example, in the massless scalar field, we have

$$
\mathscr{A}^{\mathrm{dyn}}(\boldsymbol{M} ; O)=\mathscr{A}^{\mathrm{kin}}(\boldsymbol{M} ; O) \vee \Gamma_{\odot}\left(\mathscr{L}_{\text {l.c. }}(\boldsymbol{M})\right)
$$

which differs from $\mathscr{A}^{\text {kin }}(\boldsymbol{M} ; O)$ in any spacetime with (at least one connected component having) a compact Cauchy surface. The failure of dynamical locality in this case is related to another pathology of the model, namely, the nonexistence of ground states in ultrastatic spacetimes with compact Cauchy surface. Again it is the locally constant solutions (usually regarded in terms of the zero modes of the spatial Laplacian) that create the problem, which is absent in the theory of the massless current. We observe that the rigid $\phi \mapsto \phi+$ const symmetry is spontaneously broken — it cannot be unitarily implemented even in the Minkowski vacuum state although it is an automorphism of the algebra [42]; at a higher level the symmetry generates automorphisms of the functor $\mathscr{A}[22$.

\section{Quantized theory: Weyl algebra}

In this section, we study the other main approach to the quantization of linear field theories, namely the Weyl algebra approach. We show that dynamically local theories valued in preSympl $_{\mathbb{R}}$ have dynamically local quantizations under mild additional conditions: essentially it is enough that the symplectic products of the classical theory are nondegenerate (so the theory factors via $\left.S_{y m p}\right|_{\mathbb{R}}$ ) and the relative Cauchy evolution acts continuously in a certain sense. These conditions are met by the massive Klein-Gordon theory and the theory of currents. It seems reasonable to expect that the Proca and (with some care) electromagnetic fields could also be shown dynamically local. See [25] for the algebraic approach to the quantization of the Proca field and [14 for details on its locally covariant formulation; for electromagnetism, see [20, 25, 14] for the formulation in terms of vector potentials and [16] and Appendix A of [31] treatments in terms of field strength.

We begin by recalling some relevant background. If $(S, \sigma) \in$ Sympl $_{\mathbb{R}}$, the category of weakly nondegenerate real symplectic spaces 11 the $\mathrm{CCR}$ algebra $\operatorname{CCR}(S, \sigma)$ may be defined as $C^{*}$-subalgebra of the bounded linear operators on $\ell^{2}(S)$ generated by operators $\{W(u): u \in S\}$ with action $(W(u) f)(v)=e^{i \sigma(u, v) / 2} f(u+v)\left(f \in \ell^{2}(S)\right)$. These operators evidently obey the Weyl relations

$$
W(0)=1, \quad W(u)^{*}=W(-u), \quad W(u+v)=e^{i \sigma(u, v) / 2} W(u) W(v) .
$$

We write $\mathcal{W}(S, \sigma)$ for the *-algebraic span of the $W(u)$ (which simply amounts to their linear span given the Weyl relations). Accordingly, any $A \in \operatorname{CCR}(S, \sigma)$ may be written as a limit (in operator norm)

$$
A=\lim _{n \rightarrow \infty} \sum_{u \in S} a_{n}(u) W(u)
$$

\footnotetext{
${ }^{11}$ See [6] for the construction of the Weyl algebra over pre-symplectic spaces.
} 
where, for each $n$, at most finitely many $a_{n}(u)$ are nonzero, and there are at most countably many $u$ for which there is any $n$ with $a_{n}(u) \neq 012$ Considering matrix elements, it is clear that we have limits $a(u)=\lim _{n} a_{n}(u)$ for each $u$, at most countably many of which are nonzero. Let $e_{u}$ be the basis vector in $\ell^{2}(S)$ labelled by $u \in S: e_{u}(v)=\delta_{u v}$. Then

$$
A e_{0}=\lim _{n} \sum_{u \in S} a_{n}(-u) e_{u}=\sum_{u \in S} a(-u) e_{u}
$$

and $\sum_{u \in S}|a(u)|^{2}=\left\|A e_{0}\right\|^{2}<\infty$.

As is well-known, CCR is a functor from Sympl $\mathbb{R}_{\mathbb{R}}$ to $\mathrm{C}^{*}$-Alg (see, e.g., BFV or Sec. 4.2 of [4]). Accordingly, if $\mathscr{L}$ is any weakly nondegenerate theory $\mathscr{L}:$ Loc $\rightarrow$ preSympl $\mathbb{R}_{\mathbb{R}}$, then we obtain the Weyl-algebra quantization of this theory as the functor $\mathscr{W}:$ Loc $\rightarrow C^{*}$-Alg given by $\mathscr{W}=\mathrm{CCR} \circ \mathscr{L}$ (slightly abusing notation by regarding $\mathscr{L}$ as being valued in Sympl $\left.\right|_{\mathbb{R}}$, rather than preSympl $\mathbb{R}_{\mathbb{R}}$.

We will need some information concerning fixed-points. If $\alpha$ is an automorphism of $\operatorname{CCR}(S, \sigma)$ induced by symplectic automorphism $g$ of $(S, \sigma)$ then

$$
\alpha A=\lim _{n \rightarrow \infty} \sum_{u \in S} a_{n}(u) W(g u)=\lim _{n \rightarrow \infty} \sum_{u \in S} a_{n}\left(g^{-1} u\right) W(u) .
$$

Thus if $\alpha A=A$, we have in particular (by considering the action on $e_{0}$ ) that $a(u)=a\left(g^{-1} u\right.$ ) for all $u$. As $\sum_{u \in S}|a(u)|^{2}$ is finite, it follows that $a(u)$ can be nonzero only for $u$ such that $g^{k} u=u$ for some $k \in \mathbb{Z}$. Thus the fixed-point subalgebra $\operatorname{CCR}(S, \sigma)^{\alpha}$ is the closed linear span (in $B\left(\ell^{2}(S)\right)$ ) of elements of the form $\sum_{j=0}^{k-1} W\left(g^{j} u\right)$ for $g^{k} u=u$.

More generally, if we consider the fixed-point subalgebra relative to a group of automorphisms $G$ of $(S, \sigma)$ we must restrict to elements of form $\sum_{v \in G u} W(v)$ for $u$ such that the orbit $G u$ of $u$ under $G$ is finite.

Proposition 6.1 Let $I \subset \mathbb{R}$ be an open interval containing the origin, and let $(g(s))_{s \in I}$ be a family of automorphisms of $(S, \sigma)$, with $g(0)=\mathrm{id}_{(S, \sigma)}$, generating a subgroup $G \subset$ $\operatorname{Aut}(S, \sigma)$. If there is any Hausdorff topology on $S$ for which $s \mapsto g(s)$ acts continuously on $S$, then

$$
\operatorname{CCR}(S, \sigma)^{G}=\operatorname{cl} \operatorname{span}\{W(u): u \in S, g(s) u=u \forall s \in I\} .
$$

Proof: The inclusion of the right-hand side in the left is obvious. On the other hand, the remarks above show imply that $\operatorname{CCR}(S, \sigma)^{G}$ is generated by (at most) those $W(u)$ for which $\{g(s) u: s \in I\}$ is a finite set. But as there is a Hausdorff topology on $S$ so that $s \mapsto g(s) u$ is continuous, it follows that $g(s) u$ is constant and therefore equal to $g(0) u=u$ for all $s \in I$.

Theorem 6.2 Let $\mathscr{L}:$ Loc $\rightarrow$ preSympl $_{\mathbb{R}}$ be any weakly nondegenerate dynamically local theory. Suppose that for each $\boldsymbol{f} \in \operatorname{Sym}(\boldsymbol{M}), s \mapsto \operatorname{rce}_{\boldsymbol{M}}^{(\mathscr{L})}[s \boldsymbol{f}]$ acts continuously in the weak symplectic topology on some open neighbourhood of $s=0$. Then $\mathscr{W}=\mathrm{CCR} \circ \mathscr{L}: \operatorname{Loc} \rightarrow$ $\mathrm{C}^{*}$-Alg is dynamically local.

\footnotetext{
${ }^{12}$ The point is that $A$ can be realised as a limit of a sequence in $\mathcal{W}(S, \sigma)$, each term of which involves only finitely many $u \in S$. Thus at most countably many such $u$ appear in this sequence as a whole.
} 
Proof: Let $\boldsymbol{M} \in$ Loc be arbitrary, and $O \in \mathscr{O}(\boldsymbol{M})$ be nonempty. We first observe that $\mathscr{W}^{\text {kin }}(\boldsymbol{M} ; O)$ is generated by Weyl generators $W_{\boldsymbol{M}}(u)$ indexed by $u \in \mathscr{L}^{\text {kin }}(\boldsymbol{M} ; O)$. Next, the weak symplectic topology separates points on $\left(\mathscr{L}(\boldsymbol{M}), \sigma_{\boldsymbol{M}}\right)$, because $\sigma_{\boldsymbol{M}}$ is weakly nondegenerate, and therefore defines a Hausdorff locally convex topology on $S$ 13 By Prop. 6.1,

$$
\mathscr{W}^{\bullet}(\boldsymbol{M} ; K)=\mathrm{cl} \operatorname{span}\left\{W(u): u \in \mathscr{L}^{\bullet}(\boldsymbol{M} ; K)\right\} \subset \mathscr{W}^{\mathrm{kin}}(\boldsymbol{M} ; O)
$$

because $\mathscr{L}^{\bullet}(\boldsymbol{M} ; K) \subset \mathscr{L}^{\text {kin }}(\boldsymbol{M} ; O)$ by dynamical locality of $\mathscr{L}$. Taking the $C^{*}$-union over $K \in \mathscr{K}_{b}(\boldsymbol{M} ; O)$, we find $\mathscr{W}^{\mathrm{dyn}}(\boldsymbol{M} ; O) \subset \mathscr{W}^{\text {kin }}(\boldsymbol{M} ; O)$.

On the other hand, let $u \in \mathscr{L}^{\text {kin }}(\boldsymbol{M} ; O)$. Using dynamical locality of $\mathscr{L}$, we have

$$
u \in \mathscr{L}^{\text {dyn }}(\boldsymbol{M} ; O)=\bigvee_{K \in \mathscr{K}_{b}(\boldsymbol{M} ; O)} \mathscr{L}^{\bullet}(\boldsymbol{M} ; K)
$$

so $u$ may be expressed as a finite sum $u=\sum_{i} u_{i}$, with $u_{i} \in \mathscr{L}^{\bullet}\left(\boldsymbol{M} ; K_{i}\right)$ and with $K_{i} \in$ $\mathscr{K}_{b}(\boldsymbol{M} ; O)$. Hence the Weyl generator $W_{\boldsymbol{M}}(u)$ may be expressed as a finite product of elements in the subalgebras $\mathscr{W} \bullet\left(M ; K_{i}\right)$ and is, in particular, contained in $\mathscr{W}^{\text {dyn }}(\boldsymbol{M} ; O)$. As $\mathscr{W}^{\text {kin }}(\boldsymbol{M} ; O)$ is generated by Weyl generators of this type, we have $\mathscr{W}^{\text {kin }}(\boldsymbol{M} ; O) \subset$ $\mathscr{W}^{\text {dyn }}(\boldsymbol{M} ; O)$, which concludes the proof of dynamical locality for $\mathscr{W}$.

Corollary 6.3 The Weyl algebra quantizations of (a) the Klein-Gordon theory for $m>0$ in any dimension $n \geq 2$, as a theory on $\mathrm{Loc}_{\text {or }} \mathrm{Loc}_{0}$, and (b) the theory of the massless current in any dimension $n \geq 2$ as a theory on Loc $_{0}$, or $n \geq 3$ as a theory on Loc, are dynamically local. The Weyl algebra quantizations of (c) the massless Klein-Gordon theory in any dimension $n \geq 2$, and as a theory on either $\operatorname{Loc}_{0}$ or Loc, and (d) the massless current in dimension $n=2$ as a theory on Loc, are not dynamically local.

Proof: We need only add that in the case of the massless field, the arguments above show that $\mathscr{W}^{\mathrm{dyn}}(\boldsymbol{M} ; O)$ contains $\mathscr{W}^{\text {kin }}(\boldsymbol{M} ; O) \vee \mathscr{W}_{\text {l.c. }}(\boldsymbol{M})$, where $\mathscr{W}_{\text {l.c. }}(\boldsymbol{M})$ is the subalgebra of $\mathscr{W}(\boldsymbol{M})$ generated by solutions in $\mathscr{L}_{\text {l.c. }}(\boldsymbol{M})$.

\section{Conclusion}

We have shown that the class of dynamically local theories contains at least the basic examples of free quantum fields, with the caveat that the massless Klein-Gordon field should be formulated as the theory of the massless current. Our results were obtained by first showing that our theories of interest are dynamically local as classical symplectic theories and then by applying general theorems which lift dynamical locality to the quantized theory. The existence of these theorems illustrates again the natural nature of the dynamical locality definition.

\footnotetext{
${ }^{13}$ We follow the definitions of $[39$, Ch. V] .
} 
We should like to make some comments about related work. In [9], the authors start from an (anti-)unitary representation $u$ of the proper Poincaré group in $1+d$ dimensions on a Hilbert space $\mathcal{H}$ (later interpreted as a "one-particle space"). They consider the wedge-region $\mathcal{W}=\left\{\left(x^{0}, x^{1}, \ldots, x^{d}\right): x^{1}>0,-x^{1}<x^{0}<x^{1}\right\}$ in $1+d$ dimensional Minkowski spacetime, and the associated wedge-reflection symmetry $r_{\mathcal{W}}$ : $\left(x^{0}, x^{1}, x^{2}, \ldots, x^{d}\right) \mapsto\left(-x^{0},-x^{1}, x^{2}, \ldots, x^{d}\right)$ as well as the one-parametric group $\Lambda_{\mathcal{W}}(t)$ $(t \in \mathbb{R})$ of Lorentz boosts leaving the region $\mathcal{W}$ invariant. Setting $\delta_{\mathcal{W}}^{1 / 2}=u\left(\Lambda_{\mathcal{W}}(i)\right)$ and $j_{\mathcal{W}}=u\left(r_{\mathcal{W}}\right)$, they define a "one-particle Tomita operator" $s_{\mathcal{W}}=j_{\mathcal{W}} \delta_{\mathcal{W}}^{1 / 2}$, and a one-particle subspace

$$
\mathcal{K}_{\mathcal{W}}=\left\{\xi \in \operatorname{dom}\left(\delta_{\mathcal{W}}^{1 / 2}\right): s_{\mathcal{W}} \xi=\xi\right\} \subset \mathcal{H} .
$$

This is regarded as the one-particle subspace of $\mathcal{H}$ consisting of one-particle wave functions localized in $\mathcal{W}$, in the spirit of a "reverse interpretation" of the Bisognano-Wichmann theorem [30]. Passing to the second quantization on the Fock space $\mathcal{F}_{\odot}(\mathcal{H})$, they associate the von Neumann algebras $\mathcal{R}(\mathcal{W})=\left\{W(\xi): \xi \in \mathcal{K}_{\mathcal{W}}\right\}^{\prime \prime}$ with the spaces $\mathcal{K}_{\mathcal{W}}$, where $W(\xi)$ is the Weyl-operator of $\xi \in \mathcal{H}$ on $\mathcal{F}_{\odot}(\mathcal{H})$. By forming local von Neumann algebras of observables

$$
\mathcal{R}(\mathcal{O})=\bigcap_{L \mathcal{W} \supset \mathcal{O}} \mathcal{R}(L \mathcal{W})
$$

where $\mathcal{O}$ is a double cone, and $L \mathcal{W}$ is the image of $\mathcal{W}$ under any Poincaré transform $L$, one obtains a net of local observable algebras complying with the Haag-Kastler axioms, under mild, generic additional assumptions (most importantly, positivity of the energy).

The definition of $\mathcal{K}_{\mathcal{W}}$-inspired by the Bisognano-Wichmann theorem-is, in some ways, analogous to our requirement of invariance under suitable relative Cauchy evolutions. The analogy becomes somewhat more obvious on noting that $\mathcal{K}_{\mathcal{W}}$ could equivalently be characterized as the symplectic complement, in $\mathcal{H}$, of $\mathcal{K}_{\mathcal{W}^{\prime}}$, where $\mathcal{W}^{\prime}=r_{\mathcal{W}}(\mathcal{W})$ is the causal complement wedge of $\mathcal{W}$ (cf. [9, Thm 2.5]). However, the analogy does not seem, as yet, to carry much further, since the elements of the Poincaré group (or, equivalently, the Tomita-Takesaki modular objects) act globally, whereas the relative Cauchy evolution in our setting acts locally. There is clearly room for further investigation of potential relations beyond this analogy.

To conclude, we note that the strategy developed here could be applied to other linear Bose theories (and, with modifications, to linear Fermi theories as well). For example, given any collection of weakly nondegenerate dynamically local theories $\mathscr{L}_{i}$ : Loc $\rightarrow$ preSympl $_{\mathbb{C}}$ obeying $(\mathscr{L} 1-\mathscr{L} 4)$, we may form the algebraic direct sum theory $\mathscr{L}$, with

$$
\mathscr{L}(\boldsymbol{M})=\bigoplus_{i} \mathscr{L}_{i}(\boldsymbol{M}), \quad \mathscr{L}(\psi)=\bigoplus_{i} \mathscr{L}_{i}(\psi)
$$

for any $\boldsymbol{M} \in$ Loc, and Loc morphism $\psi$. As we work with algebraic direct sums, there is no issue concerning the convergence of symplectic products etc, even when $i$ runs over an infinite index set. This is clearly a weakly nondegenerate functor to preSympl $\mathrm{C}_{\mathbb{C}}$ obeying $(\mathscr{L} 1-\mathscr{L} 4)$ because of the direct sum structure. Moreover, it is equally clear that this new theory is dynamically local. Then the theory $\mathscr{Q} \circ \mathscr{L}$ is dynamically local; similarly, we 
would have this for the Weyl algebra theory $\mathrm{CCR} \circ \mathscr{L}$, provided that the relative Cauchy evolution acts continuously in the weak symplectic topology for each $\mathscr{L}_{i}$. This establishes the dynamical locality of arbitrary multi-component minimally coupled scalar fields, with arbitrary mass spectrum [treating any zero mass components using massless current theory, and subject to the same constraints on the spacetime dimension as in Corollaries 5.4 and 6.3 .

The failure of dynamical locality for the massless current in two-dimensional spacetimes with disconnected components bears some analogy to the occurrence of topological superselection sectors in the short-distance scaling limit of the massive free scalar field on two-dimensional Minkowski spacetime discussed in the scaling algebra framework in [12. Interestingly, there is a dynamical constraint involved in the construction of scaling algebra and scaling limit, and it appears that there might be a deeper connection between dynamical locality and the occurrence of topological charges, a point worthy of further investigation.

In summary, we have established that the class of dynamically local theories contains many interesting theories; others will be studied elsewhere. Theories with pure gauge degrees of freedom will not generally be expected to satisfy dynamical locality, without further modification.

Acknowledgments We thank the organisers of the workshop 'Rigorous quantum field theory in the LHC era', held at the Erwin Schrödinger Institute, Vienna, 2011, at which this work was completed, and the ESI for financial support. CJF thanks David Hunt, Ko Sanders and Benjamin Lang for useful comments.

\section{A Support subspaces}

In the body of the paper, we made use of some simple observations on linear algebra in tensor products. Although these are presumably known, we include details for completeness. In the following, $X_{1}$ and $X_{2}$ are vector spaces of possibly infinite dimension over $\mathbb{C}$, and $\otimes$ denotes the standard tensor product of vector spaces. Recall that every bilinear map $T: X_{1} \times X_{2} \rightarrow Y$ (for $Y$ any vector space) induces a unique linear map $\tilde{T}: X_{1} \otimes X_{2} \rightarrow Y$ so that $\tilde{T}\left(x_{1} \otimes x_{2}\right)=T\left(x_{1}, x_{2}\right)$ for all $x_{i} \in X_{i}$. In particular, this gives linear maps

$$
L\left(X_{1}^{*}, X_{2}\right) \stackrel{\rho_{1}}{\longleftarrow} X_{1} \otimes X_{2} \stackrel{\rho_{2}}{\longrightarrow} L\left(X_{2}^{*}, X_{1}\right)
$$

so that, for example, $\rho_{2}$ is defined by extension of the bilinear map

$$
X_{1} \times X_{2} \ni(u, v) \mapsto v^{* *}(\cdot) u \in L\left(X_{2}^{*}, X_{1}\right),
$$

where $v^{* *}$ is the canonical embedding of $v \in X_{2}$ into $X_{2}^{* *}$. Now, any $\phi \in X_{1} \otimes X_{2}$ may be written as a finite $\operatorname{sum} \phi=\sum_{i} u_{i} \otimes v_{i}$ with nonzero $u_{i} \in X_{1}, v_{i} \in X_{2}$, so $\rho_{2}(\phi)(\cdot)=\sum_{i} v_{i}^{* *}(\cdot) u_{i}$ is a finite rank map, as is $\rho_{1}(\phi)$. Moreover, by combining and

possibly discarding terms, we may assume that the $u_{i}$ and $v_{i}$ in the expansion of $\phi$ each form linearly independent sets, which are then easily seen to span the images of $\rho_{1}(\phi)$ 
and $\rho_{2}(\phi)$ respectively (consider the applications of these maps to dual bases to the bases formed by extensions of the $u_{i}$ and $\left.v_{i}\right)$. We see then that $\phi \in \operatorname{im} \rho_{1}(\phi) \otimes \operatorname{im} \rho_{2}(\phi)$, which also proves that the $\rho_{i}$ are injective.

More generally, given any vector spaces $X_{1}, \ldots, X_{n}$, we have injections

$$
\rho_{k}: X_{1} \otimes \cdots \otimes X_{n} \rightarrow L\left(\left(X_{1} \otimes \cdots \widehat{X_{k}} \cdots \otimes X_{n}\right)^{*}, X_{k}\right)
$$

where the hat denotes an omitted factor, and any $\phi \in X_{1} \otimes \cdots \otimes X_{n}$ obeys

$$
\phi \in \operatorname{im} \rho_{1}(\phi) \otimes \cdots \otimes \operatorname{im} \rho_{n}(\phi) .
$$

We refer to the subspaces im $\rho_{k}(\phi) \subset X_{k}$ as support subspaces of $\phi$. If all the spaces $X_{1}, \ldots X_{n}$ are the same, and $\phi$ is an element of the symmetric or antisymmetric subspaces of $X^{\otimes n}$, then all the support subspaces are identical.

Lemma A.1 Suppose vector spaces $X_{1}, \ldots, X_{n}$ and $Y_{1}, \ldots, Y_{n}$ are given $(n \geq 1)$, with $S_{i} \in L\left(X_{i}, Y_{i}\right)$ for each $i$, and define $T_{n}=S_{1} \otimes \cdots \otimes S_{n}$. (a) If each $S_{i}$ is monic then $T_{n}$ is also injective. (b) If $Y_{i}=X_{i}$ for each $i$ and $\phi \neq 0$ is an eigenvector of $T_{n}$ with eigenvalue $\lambda \neq 0$, then for each $1 \leq k \leq n, S_{k}$ restricts to an automorphism of the support subspace $\operatorname{im} \rho_{k}(\phi)$.

Proof: (a) We argue by induction on $n$ : suppose this is known to be true for some $n \geq 1$ (it is true for $n=1$ ). If $T_{n+1} \phi=0$ then

$$
0=\rho_{n+1}\left(T_{n+1} \phi\right)=S_{n+1} \circ \rho_{n+1}(\phi) \circ T_{n}^{*} .
$$

Now $T_{n}$ is injective by the inductive hypothesis, so $T_{n}^{*}$ is surjective (see e.g. [28, §2.28]), while $S_{n+1}$ is injective by hypothesis. Thus $\rho_{n+1}(\phi)=0$ and thus $\phi=0$ by injectivity of $\rho_{n+1}$. Hence $T_{n+1}$ is injective and the result follows. (For a different argument, see [29, $\S 1.18]$.

For (b), we have

$$
S_{k} \circ \rho_{k}(\phi) \circ U_{k}^{*}=\rho_{k}\left(T_{n} \phi\right)=\lambda \rho_{k}(\phi)
$$

for each $k=1, \ldots, n$, where $U_{k}=S_{1} \otimes \cdots \widehat{S_{k}} \cdots \otimes S_{n}$. As $\lambda \neq 0$, it follows that $\operatorname{im} \rho_{k}(\phi) \subset$ $S_{k}\left(\operatorname{im} \rho_{k}(\phi)\right)$, which, on dimension-counting grounds, is possible only if $S_{k}$ restricts to an isomorphism of the support space $\operatorname{im} \rho_{k}(\phi)$ with itself.

\section{B Differentiability of classical relative Cauchy evolution}

We indicate how differentiability of $\operatorname{rce}_{\boldsymbol{M}}^{\mathscr{L}_{\mathbb{K}}}$ may be established in the weak symplectic topology for $\mathbb{K}=\mathbb{R}, \mathbb{C}$. By convention, we take spaces of smooth functions to be $\mathbb{K}$-valued. Fix a relatively compact open set $O \subset \boldsymbol{M}$ and Cauchy surfaces $\Sigma_{i}(1 \leq i \leq 4)$ with $\Sigma_{i+1} \subset I_{\boldsymbol{M}}^{+}\left(\Sigma_{i}\right)$ for $i=1,2,3$ and $\operatorname{cl}(O) \subset I_{\boldsymbol{M}}^{+}\left(\Sigma_{2}\right) \cap I_{\boldsymbol{M}}^{-}\left(\Sigma_{3}\right)$. In general, we write $\boldsymbol{M}_{i, j}$ for $I_{\boldsymbol{M}}^{+}\left(\Sigma_{i}\right) \cap I_{\boldsymbol{M}}^{-}\left(\Sigma_{j}\right)$. To start with, let $\boldsymbol{h} \in H(\boldsymbol{M} ; O)$ be any hyperbolic perturbation supported in $O$. 
Owing to the time-slice property, it suffices to study the action of rce $_{\boldsymbol{M}}[\boldsymbol{h}]$ on $E_{\boldsymbol{M}} C_{0}^{\infty}\left(\boldsymbol{M}_{3,4}\right)$. Fix $\chi \in C^{\infty}(\boldsymbol{M})$, with $\chi=0$ on $I_{\boldsymbol{M}}^{+}\left(\Sigma_{2}\right)$ and $\chi=1$ on $I_{\boldsymbol{M}}^{-}\left(\Sigma_{1}\right)$. Then the identity

$$
\operatorname{rce}_{\boldsymbol{M}}[\boldsymbol{h}] E_{M}=E_{M} P_{M} \chi E_{M[\boldsymbol{h}]}=E_{M} P_{M} \chi E_{M[\boldsymbol{h}]}^{-}
$$

holds on $C_{0}^{\infty}\left(\boldsymbol{M}_{3,4}\right)$. Next, the identity $P_{\boldsymbol{M}} E_{\boldsymbol{M}[\boldsymbol{h}]}^{-} f=f+\left(P_{\boldsymbol{M}}-P_{\boldsymbol{M}[\boldsymbol{h}]}\right) E_{\boldsymbol{M}[\boldsymbol{h}]}^{-} f$ entails (as both terms on the right-hand side are compactly supported)

$$
E_{M[h]}^{-} f=E_{M}^{-} f-E_{M}^{-} K_{M[h]} E_{M[h]}^{-} f
$$

by uniqueness of advanced solutions to the inhomogeneous equation, where $K_{M[h]}=$ $P_{M[h]}-P_{M}$. Iterating this formula,

$$
E_{M[h]}^{-} f=E_{M}^{-} f-E_{M}^{-} K_{\boldsymbol{M}[\boldsymbol{h}]} E_{\boldsymbol{M}}^{-} f+E_{\boldsymbol{M}}^{-} K_{\boldsymbol{M}[\boldsymbol{h}]} E_{\boldsymbol{M}}^{-} K_{\boldsymbol{M}[\boldsymbol{h}]} E_{\boldsymbol{M}[\boldsymbol{h}]}^{-} f
$$

for all $f \in C_{0}^{\infty}(\boldsymbol{M})$. Substituting this in (B.1), we have

$$
\left(\mathrm{rce}_{\boldsymbol{M}}[\boldsymbol{h}]-\mathrm{id}\right) E_{\boldsymbol{M}} f=-E_{\boldsymbol{M}} P_{\boldsymbol{M}} \chi E_{\boldsymbol{M}}^{-} K_{\boldsymbol{M}[\boldsymbol{h}]}\left(E_{\boldsymbol{M}}^{-} f-E_{\boldsymbol{M}}^{-} K_{\boldsymbol{M}[\boldsymbol{h}]} E_{\boldsymbol{M}[\boldsymbol{h}]}^{-} f\right)
$$

for $f \in C_{0}^{\infty}\left(\boldsymbol{M}_{3,4}\right)$. Now as supp $\chi$ lies to the past of the support of $K_{\boldsymbol{M}[\boldsymbol{h}]} \phi$ for any smooth $\phi$, we may replace $\chi E_{M}^{-}$by $\chi E_{M}$ in the above formula, and use the fact that $E_{M} P_{M} \chi E_{M}=E_{M}$. Thus

$$
\begin{aligned}
\left(\operatorname{rce}_{\boldsymbol{M}}[\boldsymbol{h}]-\mathrm{id}\right) E_{\boldsymbol{M}} f & =-E_{\boldsymbol{M}} K_{\boldsymbol{M}[\boldsymbol{h}]}\left(E_{\boldsymbol{M}}^{-} f-E_{\boldsymbol{M}}^{-} K_{\boldsymbol{M}[\boldsymbol{h}]} E_{\boldsymbol{M}[\boldsymbol{h}]}^{-} f\right) \\
& =-E_{\boldsymbol{M}} K_{\boldsymbol{M}[\boldsymbol{h}]} E_{\boldsymbol{M}} f+E_{\boldsymbol{M}} K_{\boldsymbol{M}[\boldsymbol{h}]} E_{\boldsymbol{M}}^{-} K_{\boldsymbol{M}[\boldsymbol{h}]} E_{\boldsymbol{M}[\boldsymbol{h}]}^{-} f .
\end{aligned}
$$

Taking symplectic products with $\phi^{\prime} \in \mathscr{L}_{\mathbb{K}}(\boldsymbol{M})$, we obtain

$\sigma_{\boldsymbol{M}}\left(\left(\mathrm{rce}_{\boldsymbol{M}}[\boldsymbol{h}]-\mathrm{id}\right) E_{\boldsymbol{M}} f, \phi^{\prime}\right)=-\int_{\boldsymbol{M}} \phi^{\prime} K_{\boldsymbol{M}[\boldsymbol{h}]} E_{\boldsymbol{M}} f d \mathrm{vol}_{\boldsymbol{M}}+\int_{\boldsymbol{M}} \phi^{\prime} K_{\boldsymbol{M}[\boldsymbol{h}]} E_{\boldsymbol{M}}^{-} K_{\boldsymbol{M}[\boldsymbol{h}]} E_{\boldsymbol{M}[\boldsymbol{h}]}^{-} f d \mathrm{vol}_{\boldsymbol{M}}$.

Now put $\boldsymbol{h}=s \boldsymbol{f}$ for $\boldsymbol{f} \in \operatorname{Sym}(\boldsymbol{M} ; O)$. We will argue below that the second integral in the previous formula is of order $O\left(s^{2}\right)$ as $s \rightarrow 0$, with $\phi^{\prime}$ and $f$ fixed. Accordingly,

$$
\begin{aligned}
\left.\frac{d}{d s} \sigma_{M}\left(\mathrm{rce}_{\boldsymbol{M}}[\boldsymbol{h}] \phi, \phi^{\prime}\right)\right|_{s=0} & =-\lim _{s \rightarrow 0} \frac{1}{s} \int_{\boldsymbol{M}} \phi^{\prime} K_{\boldsymbol{M}[s]} \phi d \mathrm{vol}_{M} \\
& =\int_{\boldsymbol{M}} \phi^{\prime}\left(\frac{1}{2}\left(\nabla^{a} f^{b}{ }_{b}\right) \nabla_{a} \phi-\nabla_{a} f^{a b} \nabla_{b} \phi\right) d \mathrm{vol}_{M} \\
& =\sigma_{\boldsymbol{M}}\left(F_{\boldsymbol{M}}[\boldsymbol{f}] \phi, \phi^{\prime}\right),
\end{aligned}
$$

where $F_{M}[\boldsymbol{f}]$ is as defined in the body of the paper.

To complete the proof we need to establish the $O\left(s^{2}\right)$ behaviour for the integral given above. The integrand is supported in $\boldsymbol{M}_{2,4}$, on which both $\phi^{\prime}$ and $K_{\boldsymbol{M}[\boldsymbol{h}]} E_{\boldsymbol{M}}^{-} K_{\boldsymbol{M}[\boldsymbol{h}]} E_{\boldsymbol{M}[\boldsymbol{h}]}^{-} f$ 
are square-integrable. It is therefore enough to show that the $L^{2}$-norm of the latter is $O\left(s^{2}\right)$ as $s \rightarrow 0$. This may be done by means of energy estimates, which show that

$$
\left\|E_{\boldsymbol{M}[s \boldsymbol{f}]}^{-} f\right\|_{p} \leq C_{p}\|f\|_{p-1} \quad\left(f \in C_{0}^{\infty}\left(\boldsymbol{M}_{2,4}\right)\right)
$$

for any $p \geq 1$, with $C_{p}$ uniform in $s$ for sufficiently small $s$, and where $\|\cdot\|_{p}$ is an energy norm on the strip $\boldsymbol{M}_{2,4}$. (See, e.g., the proof of Thm 3.7 of [13, Appx 3], noting that the condition of Sobolev regularity holds as we are able to work within a finite number of charts.) Thus we have

$$
\left\|K_{\boldsymbol{M}[s \boldsymbol{f}]} E_{\boldsymbol{M}}^{-} K_{\boldsymbol{M}[s \boldsymbol{f}]} E_{\boldsymbol{M}[\boldsymbol{f}]}^{-} f\right\|_{0} \leq C_{2} C_{3}\left\|K_{\boldsymbol{M}[s \boldsymbol{f}]}\right\|_{0,2}\left\|K_{\boldsymbol{M}[s \boldsymbol{f}]}\right\|_{1,3}\|f\|_{2}
$$

and the required estimate follows because the first two norms on the right-hand side are each of order $O(s)$ as $s \rightarrow 0$.

\section{References}

[1] Abraham, R., Marsden, J.E., Ratiu, T.: Manifolds, tensor analysis, and applications, Applied Mathematical Sciences, vol. 75, second edn. Springer-Verlag, New York (1988)

[2] Adámek, J., Herrlich, H., Strecker, G.E.: Abstract and concrete categories: the joy of cats. Repr. Theory Appl. Categ. pp. 1-507 (2006), reprint of the 1990 original [Wiley, New York]

[3] Baez, J.C., Segal, I.E., Zhou, Z.F.: Introduction to algebraic and constructive quantum field theory. Princeton Series in Physics. Princeton University Press, Princeton, NJ (1992)

[4] Bär, C., Ginoux, N., Pfäffle, F.: Wave equations on Lorentzian manifolds and quantization. European Mathematical Society (EMS), Zürich (2007)

[5] Beem, J.K., Ehrlich, P.E., Easley, K.L.: Global Lorentzian geometry, Monographs and Textbooks in Pure and Applied Mathematics, vol. 202, second edn. Marcel Dekker Inc., New York (1996)

[6] Binz, E., Honegger, R., Rieckers, A.: Construction and uniqueness of the $C^{*}$-Weyl algebra over a general pre-symplectic space. J. Math. Phys. 45, 2885-2907 (2004)

[7] Brunetti, R., Fredenhagen, K.: Microlocal analysis and interacting quantum field theories: Renormalization on physical backgrounds. Commun. Math. Phys. 208, 623661 (2000)

[8] Brunetti, R., Fredenhagen, K., Verch, R.: The generally covariant locality principle: A new paradigm for local quantum physics. Commun. Math. Phys. 237, 31-68 (2003)

[9] Brunetti, R., Guido, D., Longo, R.: Modular localization and Wigner particles. Rev. Math. Phys. 14, 759-785 (2002)

[10] Brunetti, R., Ruzzi, G.: Superselection sectors and general covariance. I. Commun. Math. Phys. 270, 69-108 (2007) 
[11] Brunetti, R., Ruzzi, G.: Quantum charges and spacetime topology: The emergence of new superselection sectors. Commun. Math. Phys. 287, 523-563 (2009)

[12] Buchholz, D., Verch, R.: Scaling algebras and renormalization group in algebraic quantum field theory. II. Instructive examples. Rev. Math. Phys. 10, 775-800 (1998)

[13] Choquet-Bruhat, Y.: General relativity and the Einstein equations. Oxford Mathematical Monographs. Oxford University Press, Oxford (2009)

[14] Dappiaggi, C.: Remarks on the Reeh-Schlieder property for higher spin free fields on curved spacetimes. Rev. Math. Phys. 23, 1035-1062 (2011), arXiv:1102.5270

[15] Dappiaggi, C., Fredenhagen, K., Pinamonti, N.: Stable cosmological models driven by a free quantum scalar field. Phys. Rev. D77, 104015 (2008)

[16] Dappiaggi, C., Lang, B.: Quantization of Maxwell's equations on curved backgrounds and general local covariance (2011), arXiv:1104.1374

[17] Degner, A., Verch, R.: Cosmological particle creation in states of low energy. J. Math. Phys. 51, 022302 (2010)

[18] Dikranjan, D., Tholen, W.: Categorical structure of closure operators, Mathematics and its Applications, vol. 346. Kluwer Academic Publishers Group, Dordrecht (1995)

[19] Dimock, J.: Algebras of local observables on a manifold. Commun. Math. Phys. 77, 219-228 (1980)

[20] Dimock, J.: Quantized electromagnetic field on a manifold. Rev. Math. Phys. 4, 223-233 (1992)

[21] Ferguson, M.: Dynamical locality of the nonminimally coupled scalar field and enlarged algebra of Wick polynomials. arXiv:1203.2151

[22] Fewster, C.J.: Endomorphisms and automorphisms of locally covariant quantum field theories. arXiv:1201.3295

[23] Fewster, C.J.: Quantum energy inequalities and local covariance. II. Categorical formulation. Gen. Relativity Gravitation 39, 1855-1890 (2007)

[24] Fewster, C.J.: On the notion of 'the same physics in all spacetimes'. In: F. Finster, O. Müller, M. Nardmann, J. Tolksdorf, E. Zeidler (eds.) Quantum Field Theory and Gravity. Conceptual and mathematical advances in the search for a unified framework. Birkhäuser (2012). arXiv:1105.6202

[25] Fewster, C.J., Pfenning, M.J.: A quantum weak energy inequality for spin-one fields in curved spacetime. J. Math. Phys. 44, 4480-4513 (2003)

[26] Fewster, C.J., Pfenning, M.J.: Quantum energy inequalities and local covariance. I: Globally hyperbolic spacetimes. J. Math. Phys. 47, 082303 (2006)

[27] Fewster, C.J., Verch, R.: Dynamical locality and covariance: What makes a physical theory the same in all spacetimes? arXiv:1106.4785, to appear in Annales H. Poincaré

[28] Greub, W.H.: Linear algebra. Third edition. Die Grundlehren der Mathematischen Wissenschaften, Band 97. Springer-Verlag New York, Inc., New York (1967)

[29] Greub, W.H.: Multilinear algebra. Die Grundlehren der Mathematischen Wissenschaften, Band 136. Springer-Verlag New York, Inc., New York (1967)

[30] Haag, R.: Local Quantum Physics: Fields, Particles, Algebras. Springer-Verlag, Berlin (1992) 
[31] Hollands, S.: Renormalized quantum Yang-Mills fields in curved spacetime. Rev. Math. Phys. 20, 1033-1172 (2008)

[32] Hollands, S., Wald, R.M.: Local Wick polynomials and time ordered products of quantum fields in curved spacetime. Commun. Math. Phys. 223, 289-326 (2001)

[33] Hollands, S., Wald, R.M.: Existence of local covariant time ordered products of quantum fields in curved spacetime. Commun. Math. Phys. 231, 309-345 (2002)

[34] Landau, L.J.: A note on extended locality. Commun. Math. Phys. 13, 246-253 (1969)

[35] Mac Lane, S.: Categories for the Working Mathematician, 2nd edn. Springer-Verlag, New York (1998)

[36] Manuceau, J., Verbeure, A.: Quasi-free states of the C.C.R.-algebra and Bogoliubov transformations. Comm. Math. Phys. 9, 293-302 (1968)

[37] O'Neill, B.: Semi-Riemannian Geometry. Academic Press, New York (1983)

[38] Pfenning, M.J.: Quantization of the Maxwell field in curved spacetimes of arbitrary dimension. Class. Quantum Grav. 26, 135017 (2009)

[39] Reed, M., Simon, B.: Methods of modern mathematical physics. I: Functional Analysis, second edn. Academic Press Inc. [Harcourt Brace Jovanovich Publishers], New York (1980)

[40] Sanders, K.: On the Reeh-Schlieder property in curved spacetime. Commun. Math. Phys. 288, 271-285 (2009)

[41] Schoch, A.: On the simplicity of Haag fields. Int. J. Theor. Phys. 1, 107-113 (1968)

[42] Streater, R.F.: Spontaneous breakdown of symmetry in axiomatic theory. Proc. Roy. Soc. Ser. A 287, 510-518 (1965)

[43] Verch, R.: A spin-statistics theorem for quantum fields on curved spacetime manifolds in a generally covariant framework. Commun. Math. Phys. 223, 261-288 (2001)

[44] Verch, R.: Local covariance, renormalization ambiguity, and local thermal equilibrium in cosmology. In: F. Finster, O. Müller, M. Nardmann, J. Tolksdorf, E. Zeidler (eds.) Quantum Field Theory and Gravity. Conceptual and mathematical advances in the search for a unified framework. Birkhäuser (2012). arXiv:1105.6249 